\title{
Time-resolved serial femtosecond crystallography at the European XFEL
}

Suraj Pandey, 1

Richard Bean, 2

Tokushi Sato, 2

Ishwor Poudyal, 1

Johan Bielecki, 2

Jorvani Cruz Villarreal, 3

Oleksandr Yefanov, 4

Valerio Mariani, 4

Thomas A. White, 4

Christopher Kupitz, 5

Mark Hunter, 5

Mohamed H. Abdellatif, 4 
Saša Bajt, 6

Valerii Bondar, 2

Austin Echelmeier, 3

Diandra Doppler, 3

Moritz Emons, 2

Matthias Frank, 7

Raimund Fromme, 3

Yaroslav Gevorkov, 4,8

Gabriele Giovanetti, 2

Man Jiang, 2

Daihyun Kim, 3

Yoonhee Kim, 2

Henry Kirkwood, 2

Anna Klimovskaia, 2

Juraj Knoska, 4

Faisal H. M. Koua, 4

Romain Letrun, 2

Stella Lisova, 9

Luis Maia, 2

Victoria Mazalova, 4

Domingo Meza, 10 
Thomas Michelat, 2

Abbas Ourmazd, 1

Guido Palmer, 2

Marco Ramilli, 2

Robin Schubert, 10

Peter Schwander, 1

Alessandro Silenzi, 2

Jolanta Sztuk-Dambietz, 2

Alexandra Tolstikova, 4

Henry N. Chapman, 4,11,12

Alexandra Ros, 3

Anton Barty, 4

Petra Fromme, 3

Adrian P. Mancuso, 2,13

Marius Schmidt, $1 \square$

Emailsmarius@uwm.edu

1 Physics Department, University of Wisconsin-Milwaukee, Milwaukee, WI, USA

2 European XFEL GmbH, Schenefeld, Germany

3 School of Molecular Sciences, and Center for Applied Structural Discovery, The Biodesign Institute, Arizona State University, Tempe, AZ, USA

4 Center for Free-Electron Laser Science, Deutsches Elektronen Synchrotron, Hamburg, Germany 
Linac Coherent Light Source, Stanford Linear Accelerator Center, National Accelerator Laboratory, Menlo Park, CA, USA

6 Deutsches Elektronen Synchrotron, Hamburg, Germany

7 Lawrence Livermore National Laboratory, Livermore, CA, USA

8 Institute of Vision Systems, Hamburg University of Technology, Hamburg, Germany

9 Physics Department, Arizona State University, Tempe, AZ, USA

10 Integrated Biology Infrastructure Life-Science Facility at the European XFEL, Schenefeld, Germany

11 University of Hamburg, Hamburg, Germany

12 Centre for Ultrafast Imaging, Hamburg, Germany

13 Department of Chemistry and Physics, La Trobe Institute for Molecular Science, La Trobe University, Melbourne, Victoria, Australia

Received: 10 June 2019 / Accepted: 3 October 2019

\section{Abstract}

The European XFEL (EuXFEL) is a 3.4-km long X-ray source, which produces femtosecond, ultrabrilliant and spatially coherent X-ray pulses at megahertz $(\mathrm{MHz})$ repetition rates. This X-ray source has been designed to enable the observation of ultrafast processes with near-atomic spatial resolution. Time-resolved crystallographic investigations on biological macromolecules belong to an important class of experiments that explore fundamental and functional structural displacements in these molecules. Due to the unusual $\mathrm{MHz}$ X-ray pulse structure at the EuXFEL, these experiments are challenging. Here, we demonstrate how a biological reaction can be followed on ultrafast timescales at the EuXFEL. We investigate the picosecond time range in the photocycle of photoactive yellow protein (PYP) with $\mathrm{MHz}$ 
X-ray pulse rates. We show that difference electron density maps of excellent quality can be obtained. The results connect the previously explored femtosecond PYP dynamics to timescales accessible at synchrotrons. This opens the door to a wide range of time-resolved studies at the EuXFEL.

\section{Editor's Summary}

European XFEL allows investigation of the picosecond time range in the photocycle of photoactive yellow protein.

These authors contributed equally: Suraj Pandey, Richard Bean, Tokushi Sato.

\section{Main}

Time-resolved macromolecular crystallography (TRX) combines macromolecular structure determination with reaction dynamics[1,2]. Short and ultrashort light pulses are employed to enable snapshot observations that cope with the relevant timescales of biomolecular reactions. With TRX, biologically, biomedically and pharmacologically important reactions can be observed in real time with atomic or near-atomic spatial resolution. Hard X-ray free-electron lasers (XFELs) substantially changed the way TRX experiments were conducted[3, 4], a direct consequence of the unprecedented brilliance of XFELs, and their short (femtosecond) X-ray pulses. Instead of examining macroscopically large crystals, microcrystals are injected into the X-ray beam[5,6] at room temperature. Although these microcrystals are often destroyed, the femtosecond X-ray pulse duration at the XFEL largely outruns radiation damage and the associated structural rearrangements $[7,8,9,10]$. Once exposed to the XFEL beam, the crystal must be replaced, demanding a serial approach where, for each new observation, a pristine microcrystal interacts with the subsequent X-ray pulse, a technique known as serial femtosecond crystallography (SFX)[5]. It has been demonstrated recently at the EuXFEL that SFX is possible with megahertz X-ray pulses[11, 12]. In time-resolved SFX (TR-SFX) a reaction in a microcrystal is initiated with an optical laser during sample delivery into the X-ray interaction volume, and the progress of the reaction is probed after a time delay $\Delta t$ by the pulsed XFEL beam, as pioneered at the Linac Coherent Light Source (LCLS) at $120 \mathrm{~Hz}$ (refs. $[3,4,13,14]$ ). TR-SFX has the potential to take advantage of the megahertz peak rate of the European XFEL, to structurally map multiple stages 
of a reaction with a single experiment.

AQ1

AQ2

AQ3

AQ4

AQ5

AQ6

The experiments reported here examine the photocycle of PYP (Fig. 1a) using the MHz pulse structure of the EuXFEL (Supplementary Fig. 1). PYP is a bacterial photosensor, in which light triggers a reaction with several intermediates[15]. PYP is an excellent model system to establish TR-SFX at the EuXFEL, as it has been previously studied by TRX investigations at both synchrotrons and XFELs $[4,14,16,17]$. The photocycle is driven by the trans to cis isomerization of the central para-coumaric acid (pCA) chromophore[18] (Fig. 1b). In addition to being chemically highly important, its ultrafast dynamics displays similarities to other light-triggered reactions, including photoisomerization reactions in rhodopsin in the mammalian eye[19], and in other biologically significant photoreceptors such as the phytochromes[20]. Furthermore, PYP has become a prominent optogenetic tool that can be used for the spatiotemporal optical control of complex biological processes, such as neural activity[21]. The photocycle of PYP has been extensively investigated from femtoseconds to seconds[14, 17, 22]. However, the time range between 1 ps and $100 \mathrm{ps}$ has not been investigated in detail so far (Fig. 1c), with at least one more process observed by spectroscopy[23] (Fig. 1c, red arrows) for which there is currently no experimental structural evidence.

\section{Fig. 1}

The photocycle of PYP in crystals.

a, The photocycle (simplified) is initiated by blue light that excites the ground (dark) state $\mathrm{pG}$ to the electronic excited state $\mathrm{pG}^{*}$. After the trans to cis isomerization at $600 \mathrm{fs}$, several electronic ground-state intermediate states, called $\mathrm{I}_{\mathrm{T}}, \mathrm{pR}_{1}, \mathrm{pR}_{2}, \mathrm{pB}_{1}$ and $\mathrm{pB}_{2}$, are populated on various timescales until the photocycle completes. Approximate relaxation times are shown. Red dotted box: relaxations on the picosecond timescale. $\mathbf{b}$, The chemical structure of the pCA chromophore bound to the Cys 69 sulfur. The trans configuration is shown. The torsional angle 
$\varphi_{\text {tail }}$ as defined by chromophore carbon atoms $\mathrm{C}_{1}-\mathrm{C}_{2}=\mathrm{C}_{3}-\mathrm{C}_{1}$, is outlined in red. Hydrogen bonds between the pCA head and Glu 46 and Tyr 42 are marked. The rotation about the double bond as well as the head displacement at longer times are shown by arrows. c, The ultrafast timescale from 100 fs to $100 \mathrm{ps}$. Black dashed bars: time delays collected at the LCLS (Pande et al.[14]), green dashed bar: time delay collected at APS (Jung et al.[33]). Green solid bars: time delays in the 1 ps to 100 ps range (gray) as collected in this study. Red arrows: picosecond processes observed spectroscopically (Creelman et al. [23]).
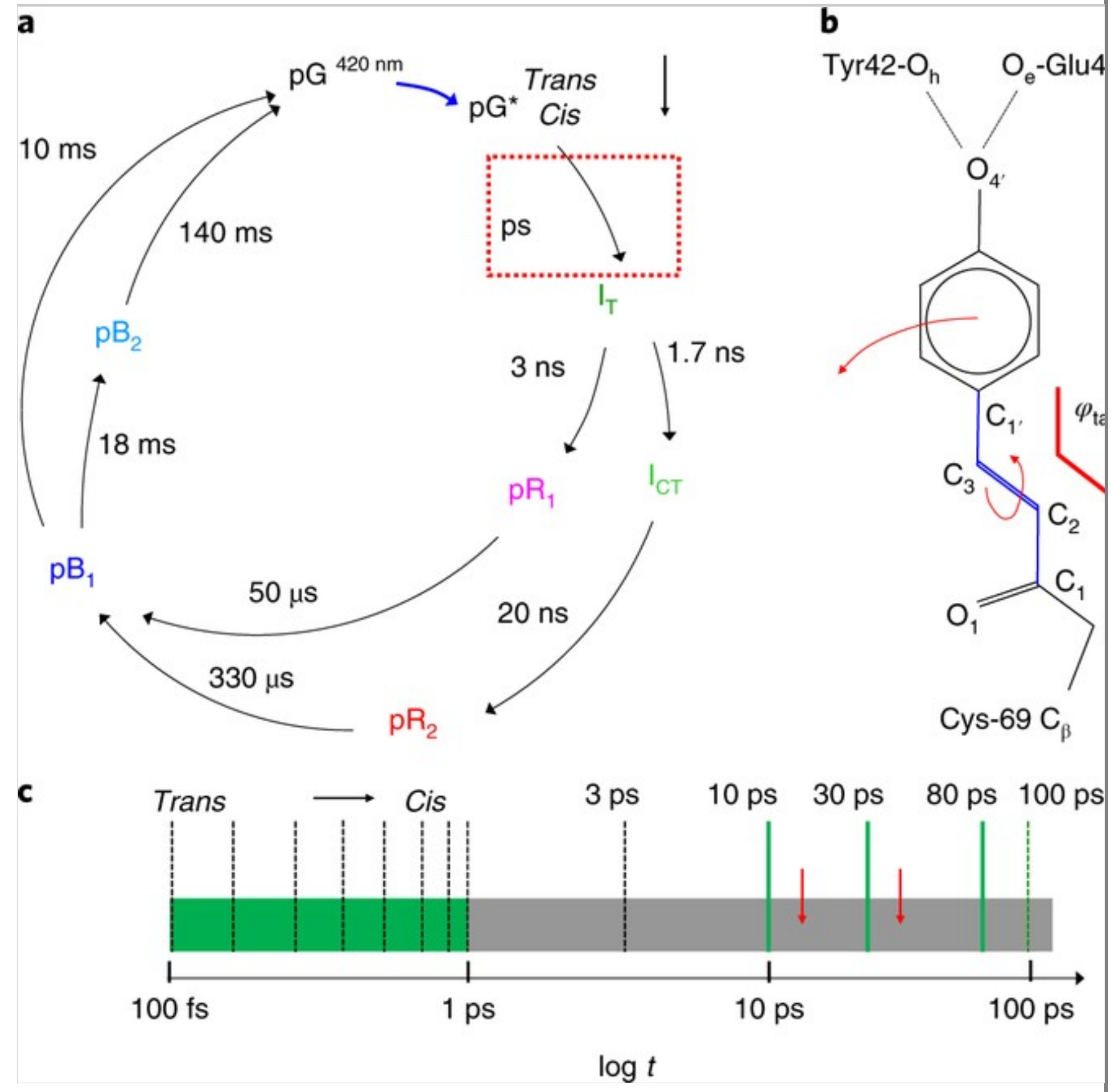
At the EuXFEL, X-rays arrive in pulse trains at $10 \mathrm{~Hz}$ (Fig. 2a and Supplementary Fig. 1). Each train consisting of bursts of X-ray pulses with an intratrain rate of up to $4.5 \mathrm{MHz}$ (ref. [11]). In the current operational configuration, each train contains up to 176 pulses at a maximum rate of 1.13 MHz. This amounts to 1,760 pulses per second - already almost 15 times more pulses per unit time than the next highest repetition rate, hard X-ray FEL. A high-intensity $\mathrm{MHz}$ optical laser system has been commissioned recently and is now available at the SPB/SFX instrument of the EuXFEL[24]. Our experiments require the synchronization of each optical laser pulse with a corresponding $\mathrm{X}$-ray pulse, in the end at $\mathrm{MHz}$ rates, and each time with picosecond precision. The high pulse repetition rate offers new opportunities for TR-SFX investigations at the EuXFEL; closely spaced time delays can be collected rapidly to cover processes in biomolecules in detail. At other XFELs, the low pulse repetition rate limits the amount of data that can be collected during sparsely available beamtimes. Additional parameters such as temperature[25], laser pulse duration and laser chirp may then be varied to control[26] and direct the biomolecular reaction.

\section{Fig. 2}

Pulse train structure and laser excitation.

a, X-ray pulse trains (black vertical lines) at EuXFEL with $1.13-\mathrm{MHz}$ pulse repetition rate. A pulse train is $156 \mu$ s long, contains $176 \mathrm{X}$-ray pulses and repeats ten times per second. There are 99.84-ms gaps between the pulse trains. Blue: laser pulses for a pump-probe dark TR-SFX data collection scheme. Note: when EuXFEL design specifications are reached, 2,700 pulses with up to $4.5 \mathrm{MHz}$ pulse repetition rate are in a train. At $4.5 \mathrm{MHz}$, each pulse train is $600 \mu$ s long with 99.4ms gaps between the trains. In total there are 27,000 pulses per second, a subset of which (about 3,520 pulses per second) can be stored in, and read out by, the AGIPD detector. b, The $1.13 \mathrm{MHz}$ control experiment with $376 \mathrm{kHz}$ laser excitation. After the laser pulse, subsequent X-ray pulses arrive at $887 \mathrm{~ns}, 1.78 \mu \mathrm{s}$ and $2.67 \mu \mathrm{s}$. The sequence repeats until the end of the pulse train. c, The $564 \mathrm{kHz}$ data collection with three interleaved X-ray pulses. There are 88 pulses in the train, only. The laser pulses are separated by $7.1 \mu \mathrm{s}(141 \mathrm{kHz})$ to provide enough time for the laser-excited volume (red) to move out of the X-ray interaction region. A total 
of 519,336 diffraction patterns were averaged to determine the scheme.

\section{a Pulse train}

(1) (2) (3) (4) $\ldots \quad$ (176)

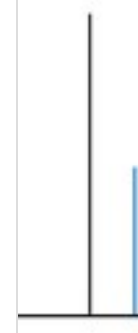

b

Control experiment at $1.13 \mathrm{MHz}$
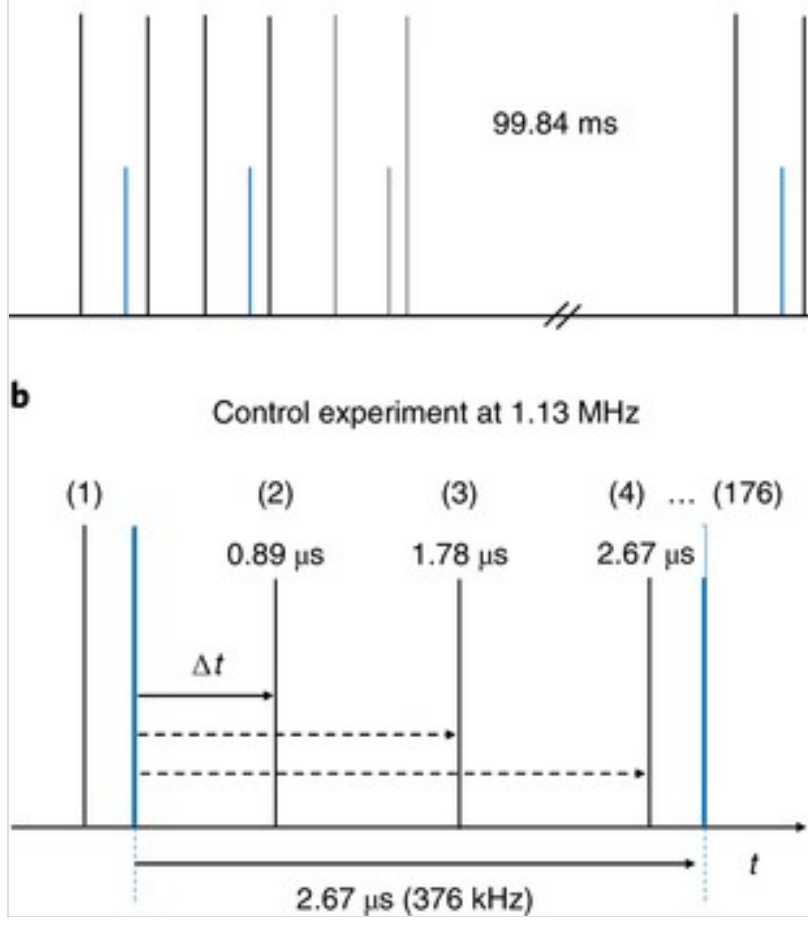

\section{$1.13 \mathrm{MHz}$}

(1) (2) (3) (4) $\ldots$ (176)
$156 \mu \mathrm{s}$

(1) (2) (3) (4) ...

(176)
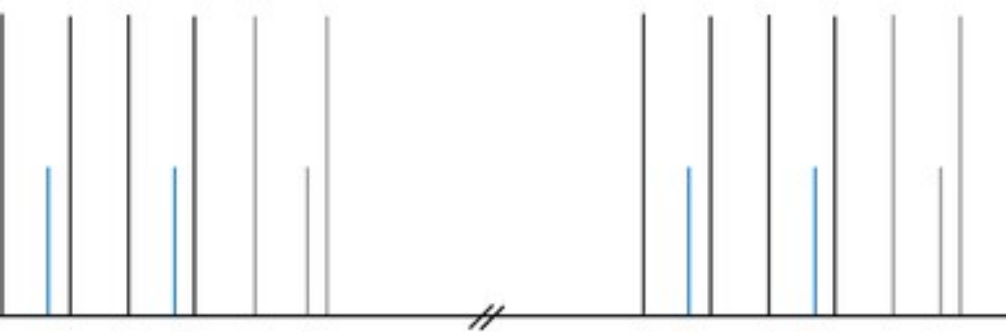

c Data collection at $564 \mathrm{kHz}$

(1)

(3) (4)

(5)

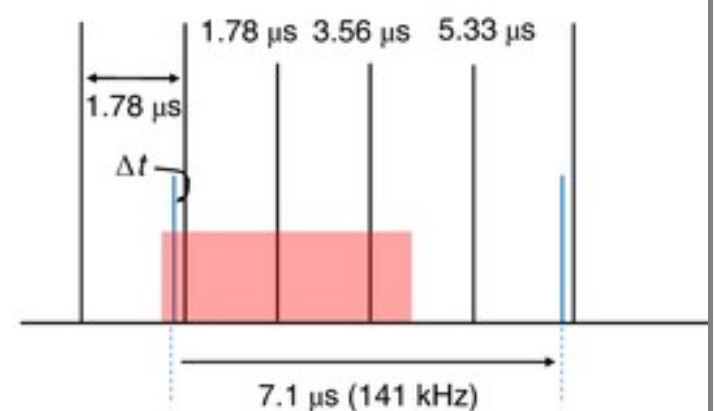

\section{Results}

\section{TR-SFX experiments}

A dense microcrystalline slurry of PYP was prepared and injected into the vacuum chamber of the SPB/SFX instrument[27] at the EuXFEL. The microcrystals were exposed to the trains of X-ray bursts. X-ray diffraction patterns were collected by the 'adaptive gain integrating pixel detector' (AGIPD)[28] operating with MHz frame rates (Supplementary Fig. 1). The PYP photocycle was initiated using laser pulses of $240 \mathrm{fs}$ at a wavelength of $420 \mathrm{~nm}$ with a flux density of $1.6 \mathrm{~mJ} \mathrm{~mm}^{-2}$ in a $42-\mu \mathrm{m}$ (full-width half maximum, FWHM) focal spot. The viscosity of the dense microcrystalline slurry placed an upper limit to the achievable jet speed of $30 \mathrm{~m} \mathrm{~s}^{-1}$ (determined in the laboratory under similar injection conditions). Initial measurements were conducted to establish optimal X-ray and laser pulse rates at the achievable jet speed. Laser pulse rates and X-ray pulse structures that were used here are shown in Fig. 2b,c.

First, we collected SFX data without any laser excitation at $1.13 \mathrm{MHz}$ X-ray 
repetition rate to establish a suitable $\mathrm{X}$-ray pulse rate to ensure that the sample was being refreshed between X-ray pulses (Supplementary Table 1, pure 'dark'). Next, we exposed crystals to the optical laser at $375 \mathrm{kHz}$ repetition rate (every third X-ray pulse; Fig. 2b, control experiment) to determine when and whether the laser-excited jet volume had passed the X-ray interaction region. Data statistics are shown in Supplementary Table 2. With a jet velocity of $30 \mathrm{~m} \mathrm{~s}^{-1}$ and a laser focus of $42 \mu \mathrm{m}$, the excited volume should leave the X-ray interaction region within $2 \mu \mathrm{s}$. Accordingly, the difference electron density (DED) map at the $2.67 \mu$ s time delay should be free of signal. However, as shown in Fig. 3, the resulting DED maps display signal at all time delays. The same density features are observed ( $\alpha$ for negative features, and $\beta 1$ and $\beta 2$ for positive features) in all difference maps. All three maps are essentially identical, and contain a mixture of PYP intermediates pR1 and pR2 that persist in the early $\mu$ s time range[17]. Compare, for example, the structure displayed in Fig. 3a determined at $1 \mu$ s delay at the LCLS[4] with those in Fig. 3b,c. In addition, the hit rate abruptly decayed from $2 \%$ at the first X-ray pulse in the train, to $1 \%$ (Supplementary Fig. 2a) in all subsequent pulses. This shows that jet velocities that are achievable with our dense PYP slurry do not reliably replace the sample at the X-ray interaction point at the $1.13 \mathrm{MHz}$ X-ray repetition rate. Consequently, both the X-ray pulse repetition rate and the laser repetition rate are too high for our planned picosecond TR-SFX experiment. However, there is no indication that there are significant structural differences caused by the $1.13 \mathrm{MHz}$ X-ray pulse rate. Values for $R / R_{\text {free }}$ values of the reference model refined against the data collected in the dark are $17 \% / 24 \%$, respectively, with no obvious differences in $F_{\text {obs }}-F_{\text {calc }}$ difference maps. It seems that, at least in the case of PYP, the crystals are not affected by acoustic shockwaves observed earlier[29] and the dominant effect is an absence of crystals at the interaction point.

Fig. 3

TR-SFX experiments at LCLS and EuXFEL.

a, DED in the PYP chromophore pocket at $1 \mu \mathrm{s}$ time delay, as determined at the LCLS (Tenboer et al.[4]). Red: negative DED, blue: positive DED on the $-3 \sigma / 3 \sigma$ contour levels, respectively. Prominent features are labeled $\alpha$ (negative) or $\beta_{1}$ and $\beta_{2}$ (positive). Features labeled $\alpha$ are on top of the reference structure (yellow), $\beta_{1}$ and $\beta_{2}$ features correspond to intermediate structures called $\mathrm{pR} 1$ (magenta) and pR2 (red), respectively. The pattern of $\alpha$ and $\beta_{1}, \beta_{2}$ features persists in all maps at 
all times. b-d, Results of the control experiment with $1.13 \mathrm{MHz}$ X-ray pulse repetition and $376 \mathrm{kHz}$ laser excitation (see also Supplementary Fig. 2b): $0.89 \mu \mathrm{s}$ after the laser pulse (b), $1.78 \mu$ s after the laser pulse (c), $2.67 \mu$ s after the laser pulse (d).
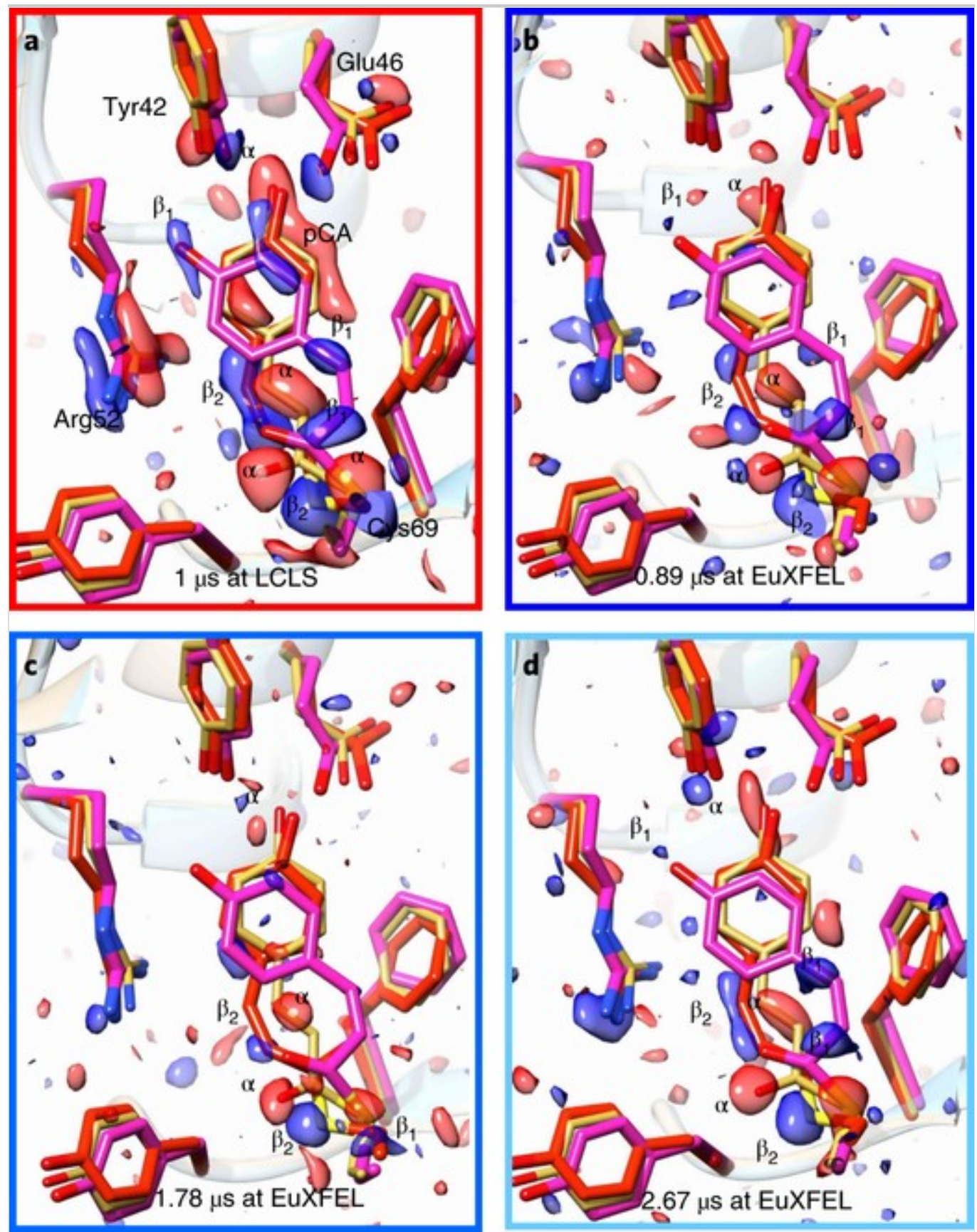

Following these observations, the X-ray repetition rate was reduced to $564 \mathrm{kHz}$ and the laser repetition rate was reduced to $141 \mathrm{kHz}$ so that laser excitation was achieved before every fourth X-ray pulse (Fig. 2c). At the $0.56 \mathrm{MHz}$ X-ray repetition rate, the hit rate across the entire pulse train remains essentially 
constant (Supplementary Fig. 2b). This shows that at $0.56 \mathrm{MHz}$ the sample is sufficiently refreshed before the next X-ray pulse arrives. The pump-probe delay was set to a value of $10 \mathrm{ps}$, with subsequent X-ray pulses measuring delays of $1.78 \mu \mathrm{s}, 3.56 \mu \mathrm{s}$ and $5.33 \mu \mathrm{s}$ (see Supplementary Table 3 for data statistics). At the 10 ps time delay, we observe a DED map with strong features (Fig. 4a) that resemble the DED map collected at a 3 ps delay at the LCLS (Fig. 5a)[14]. The DED map calculated with data from the following X-ray pulse at $1.78 \mu \mathrm{s}$ (Fig. 4b) differs completely from the $10 \mathrm{ps}$ DED map, and shows the same pattern of DED features as described in Fig. 3. It can be interpreted with the previously described mixture of intermediates with microsecond lifetimes. The microsecond DED signal decays to a spurious positive feature caused by the displacement of the electron rich Cys 69 sulfur at the $3.56 \mu$ s delay (Fig. 4c, blue arrow). This feature vanishes completely at the $5.33 \mu$ s delay, which means that a short time after $3.56 \mu$ s the laser-excited jet volume left the X-ray interaction region. Since the laser profile is Gaussian, with an FWHM of $42 \mu \mathrm{m}$, there is still substantial intensity in the flanks of the spot, which may cause contaminations up to the $3.56 \mu$ s time delay. These experiments demonstrate how the TR-SFX experiment must be set up when operating within our experimental conditions.

Fig. 4

DED and structures of the chromophore-binding region of PYP.

TR-SFX data were collected with $564 \mathrm{kHz}$ X-ray and $141 \mathrm{kHz}$ laser pulse repetition rates, respectively. a, A 10 ps time delay. Yellow: reference structure, green: $10 \mathrm{ps}$ structure. Red features $\alpha$ : negative difference DED ( $-3 \sigma$ contour level), blue, $\beta$ : positive DED ( $3 \sigma$ contour level). $\mathbf{b}$, The pattern of DED features radically changes compared with a. Magenta and red: structures of the pR1 (DED features $\beta_{1}$ ) and pR2 (DED features $\beta_{2}$ ) intermediates, respectively. c, DED after $3.56 \mu \mathrm{s}$. Only the prominent feature of the Cys 69 sulfur remains (blue arrow). d, DED at $5.33 \mu \mathrm{s}$, the feature on the Cys 69 sulfur is absent (blue arrow). DED contour levels in $\mathbf{b}-\mathbf{d}$ as in $\mathbf{a}$. 

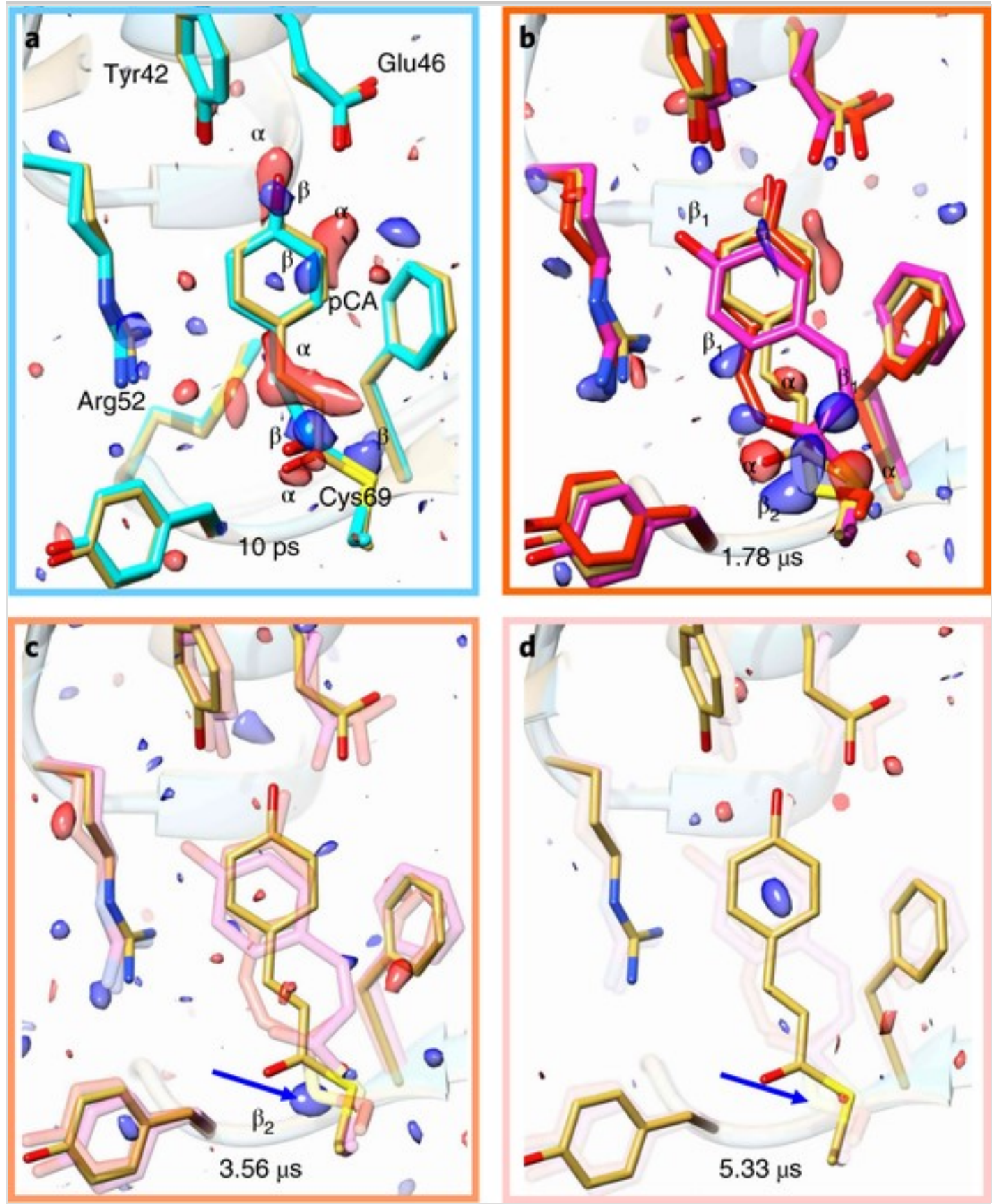

Fig. 5

Time series of TRX data from 3 ps to 100 ps collected at LCLS, EuXFEL and APS.

Structures and DED in the chromophore-binding region of PYP. Red: negative DED ( $-3 \sigma$ contour level), blue positive DED ( $3 \sigma$ contour level). Important residues and the pCA chromophore are marked in a. Yellow structure: structure of the (dark) reference state. Arrows depict structural displacements in $\mathbf{a}, \mathbf{f}$ and $\mathbf{j}$. Upper: front view, lower side view. a,f, With a 3 ps delay as collected at LCLS. Green: 
PYP structure at 3 ps (Pande et al.[14]). b,g, With a 10 ps time delay, this study, cyan: PYP structure at 10 ps. c,h, With a 30 ps time delay, this study, sky blue: PYP structure at $30 \mathrm{ps}$. d,i, With an $80 \mathrm{ps}$ time delay, this study, blue: PYP structure at $80 \mathrm{ps}$. e,j, With a $100 \mathrm{ps}$ time delay as determined at APS, light blue: PYP structure at 100 ps (Jung et al.[33]).
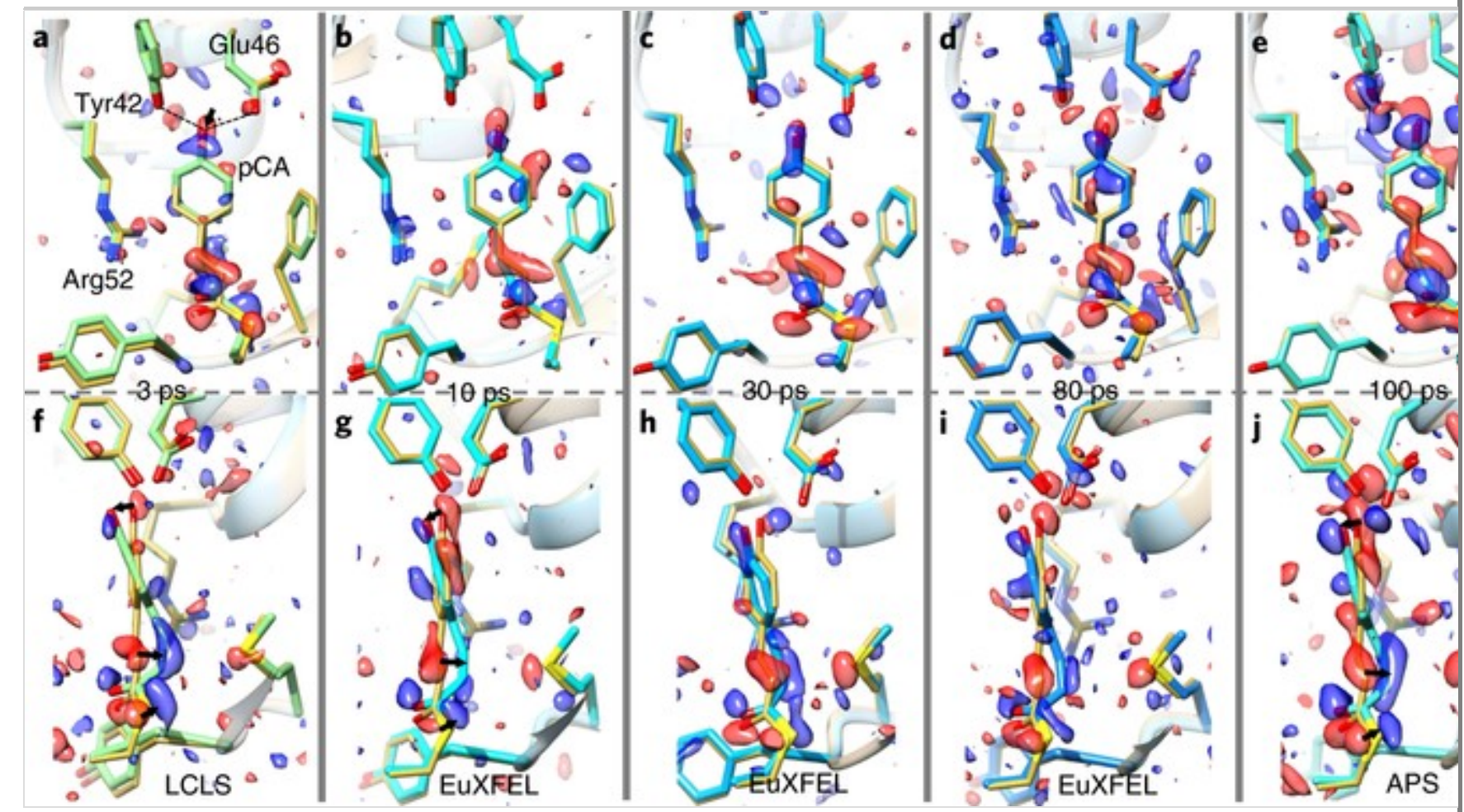

\section{Picosecond time series}

In addition to the $10 \mathrm{ps}$ time point, we collected TR-SFX datasets at $30 \mathrm{ps}$ and 80 ps time delays, with the successful timing scheme described. Fluorinated oil was added to the crystalline slurry at various ratios (Supplementary Table 1) via a T-junction (Supplementary Fig. 1). The T-junction was located close to the outside end of the nozzle rod. The oil flowed through the long capillary across the rod and was injected together with the microcrystals. In addition to a slight increase of the liquid flow rate (Supplementary Table 1), the resulting jets became substantially elongated, from $100 \mu \mathrm{m}$ to $700 \mu \mathrm{m}$. This allowed us to increase the distance from the nozzle to the interaction region to $400 \mu \mathrm{m}$, which helped to prevent the accumulation of debris on the nozzle tip that would otherwise quickly interfere with data collection. The $10 \mathrm{ps}$ to $80 \mathrm{ps}$ time delays cover the region previously unknown in the photocycle, probing a time region 
between the published LCLS data at $3 \mathrm{ps}$ and synchrotron data at $100 \mathrm{ps}$. Supplementary Table 2 lists the data statistics.

AQ9

In Fig. 5, the DED maps at $10 \mathrm{ps}, 30 \mathrm{ps}$ and 80 ps are shown together with those obtained previously at LCLS and at APS at 3 ps and 100 ps, respectively. In short, all time delays collected at the EuXFEL resulted in excellent DED maps that contain chemically meaningful positive and negative DED features ( $\alpha$ and $\beta$ in Fig. 5). The DED maps (Fig. 5b-d) are similar and comparable to those obtained at other X-ray sources on the ps timescale (Fig. 5a,e). The positive and negative DED features are interpreted by structural models using extrapolated maps[16, 30, 31, 32] (see Methods; Supplementary Tables 4 and 5 list the refinement statistics). In addition to the newly collected time delays of 10, 30 and 80 ps, we also revisited the 3 ps (ref. [14]) and the 100 ps (ref. [33]) data collected previously at LCLS and APS, respectively (Supplementary Fig. 3). We subjected all data across the time window from 3 ps to 100 ps to our objective procedures described in the Methods, to ensure consistent results.

Population transfer (PT) for each time point in this experiment is approximately $7 \%$ (see Supplementary Table 6), which is lower compared to similar excitation schemes at other XFELs. With a femtosecond laser pulse we are limited to the primary photoexcitation yield, which is $20 \%$ at best when excitation is achieved into the absorption maximum[34]. More details on how to estimate the PT are given in the Methods. The yield is further diminished here by illuminating into the flanks of the absorption spectrum: excitation was achieved at $420 \mathrm{~nm}$ rather than into the central absorption peak at $450 \mathrm{~nm}$ (Supplementary Fig. 4b). Still, excellent data can be collected because the laser penetration depth matches the micrometer crystal size[4], leading to uniform sample excitation. From the side view of the pCA chromophore at various time delays in Fig. $5 \mathrm{f}-\mathrm{j}$, one can see that the chromophore is in a twisted cis configuration throughout. The chromophore head is strongly tilted with the chromophore tail behind the plane of the unexcited (dark) pCA chromophore (see arrows in Fig. 5). Table 1 lists the torsional angle of the chromophore tail $\varphi_{\text {tail }}$ (Fig. 1b) obtained after refinement, as well as the lengths of the hydrogen bonds that fix the chromophore head to Tyr 42 and Glu 46 . The revisited $\varphi_{\text {tail }}$ at 3 ps $\left(39^{\circ}\right)$ and 100 ps $\left(30^{\circ}\right)$ agree with results published earlier $\left(35^{\circ}\right.$ and $33^{\circ}$, respectively) $[14,22]$. 


\section{Table 1}

Geometry of the pCA chromophore after refinement

\begin{tabular}{|c|c|c|c|c|c|c|}
\hline & Dark & 3 ps & $10 \mathrm{ps}$ & $30 \mathrm{ps}$ & $80 \mathrm{ps}$ & $100 \mathrm{ps}$ \\
\hline$N_{\text {ext }}^{\text {a }}$ & - & 16 & 30 & 29 & 29 & 40 \\
\hline $\begin{array}{l}\text { Hydrogen bond pCA to } \\
\text { E46 (A) }\end{array}$ & 2.55 & 3.30 & 2.82 & 2.87 & 2.86 & 2.79 \\
\hline $\begin{array}{l}\text { Hydrogen bond pCA to } \\
\text { Y42 (A) }\end{array}$ & 2.58 & 2.57 & 2.65 & 2.62 & 2.64 & 2.39 \\
\hline$\varphi_{\text {tail }}\left({ }^{\circ}\right)$ & 172 & 39 & 51 & 54 & 40 & 30 \\
\hline \multicolumn{7}{|c|}{${ }^{\mathrm{a}}$ Characteristic $N$ used to calculate extrapolated maps } \\
\hline $\begin{array}{l}\text { The length of the hydrog } \\
\text { as well as the torsional a } \\
\mathrm{C} 3 \text { and } \mathrm{C} 1^{\prime} \text { is shown. For } \\
\text { cis-configuration } \varphi \text { it is c }\end{array}$ & $\begin{array}{l}\text { bonds fro } \\
\text { e } \varphi \text { of the } \\
\text { e tail in tl } \\
\text { e to } 0^{\circ} .\end{array}$ & $\begin{array}{l}\text { the } \mathrm{pC} \\
\text { CA tail } \\
\text { trans-c }\end{array}$ & $\begin{array}{l}\text { head hy } \\
\text { efined by } \\
\text { nfigurati }\end{array}$ & $\begin{array}{l}\text { oxyl } \\
\text { CA } \\
\varphi \text { is }\end{array}$ & $\begin{array}{l}\text { lu } 46 \text { anc } \\
\text { on atoms } \\
\text { e to } 180\end{array}$ & $\begin{array}{l}\text { Tyr 46, } \\
\text { C1, C2, } \\
\text { in the }\end{array}$ \\
\hline
\end{tabular}

\section{Discussion}

\section{Structural dynamics}

When comparing results at different picosecond time delays, the torsional angle at $3 \mathrm{ps}\left(39^{\circ}\right)$ increases at $10 \mathrm{ps}\left(51^{\circ}\right)$ and $30 \mathrm{ps}\left(54^{\circ}\right)$ and relaxes through $80 \mathrm{ps}$ to a final value $\left(30^{\circ}\right)$ at $100 \mathrm{ps}$. The torsional relaxations up to 3 ps occur in concert with an initial increase of the hydrogen bond distance from the $\mathrm{pCA}^{-\mathrm{O}_{4}}$ to Glu $46-\mathrm{O}_{\mathrm{e}}(3.3 \AA)$. After $80 \mathrm{ps}$ the hydrogen bonds subsequently relax to shorter distances that approach those observed in the dark structure. Assuming that about half of the absorbed photon energy is stored in the near-cis chromophore configuration (of the order of $100 \mathrm{~kJ} \mathrm{~mol}^{-1}$ )[35], the release of the chromophore head from a network of two hydrogen bonds should be possible, since the energies of the hydrogen bonds are only about $10 \mathrm{~kJ} \mathrm{~mol}^{-1}$ (ref. [36]) each. However, for the pCA head displacements to occur, chromophore pocket relaxations are required, which are not yet developed on fast timescales. Displacements of the $M_{41-71}$ moiety (Supplementary Fig. 4a), which wraps around the chromophore pocket peak at $10 \mathrm{ps}$, revert slightly at $30 \mathrm{ps}$, and slowly increase towards 100 ps (Supplementary Fig. 4c).

The initial displacements are reminiscent of ultrafast structural dynamics 
detected by time-resolved experiments on myoglobin in solution[37]. Although the faster, ps time range (between $1 \mathrm{ps}$ and $100 \mathrm{ps}$ ) is sparsely covered by previous experiments[14, 22, 33] and the experiments at EuXFEL included here, direct structural evidence is provided to show how an energetically highly strained structure initially reorders and then only relaxes slowly for a longer period of time (Supplementary Figs. 4c and 5a,c). This is a direct visualization of a nonexponential, nonergodic, ultrafast relaxation from a high energy state towards a longer-lived thermal reservoir, which is structurally characterized by intermediate $\mathrm{I}_{\mathrm{T}}$ (Fig. 1a). Only after 100 ps, may the PYP molecules that populate this reservoir sample configurational space more comprehensively to find a reaction coordinate that permits transition to the next intermediate state. As shown previously, transitions to two intermediates $\left(\mathrm{I}_{\mathrm{CT}}\right.$ and $\left.\mathrm{pR} 1\right)$ are possible[33], requiring reaction coordinates that likely arise from two different positions in configurational space.

\section{Data collection strategy}

In this particular experimental case, reduction of the $\mathrm{X}$-ray pulse repetition rate to $564 \mathrm{kHz}$ was necessary to perform TR-SFX experiments with these dense crystalline slurries, with sample jet speeds of about $30 \mathrm{~m} \mathrm{~s}^{-1}$. The combination of jet speed and $42 \mu \mathrm{m}$ (FWHM) laser spots demanded optical laser repetition rates not faster than $141 \mathrm{kHz}$, to minimize contamination from previous exposures. In this way, 22 laser pulses are accommodated per X-ray pulse train, which amounts to 220 laser excitations per second. This rate is between a factor 3.5 and 15 times faster than that achieved previously at other XFELs.

To push data collection rates towards the $\mathrm{MHz}$ range the liquid flow rate, as well as the gas flow rate that narrows the jet to boost its speed[38], must be further increased. If synthetic oil flows together with the crystalline slurry, clogging of, and debris deposition on, the gas dynamic virtual nozzle (GDVN) is largely reduced and an increase in the flow rate is possible. With higher gas flow rates, provisions such as increased pump rates to maintain high vacuum levels are required to protect the highly sensitive $\mathrm{MHz} \mathrm{X}$-ray detector. When the laser focus is reduced to about $20 \mu \mathrm{m}$ FWHM, the laser-excited jet volume will also leave the X-ray interaction region faster. Then, $564 \mathrm{kHz}$ laser pulses interleaved by $1.13 \mathrm{MHz}$ X-ray pulses will push the speed of the pump-probe data collection rates to the limit, and a time delay can be collected of the order of minutes. Our 
results also pave the way for collecting X-ray data with femtosecond time delays at the EuXFEL. At the same time, meaningful datasets on the fast $\mu$ s timescale may be obtained, which add important time delays and may serve as invaluable controls to assess signal levels in the DED maps. When longer time delays are explored, different strategies with largely reduced laser repetition rates $(10 \mathrm{~Hz})$ may be employed, where the reaction is initiated already within the nozzle capillary and probed, after injection, by the entire train of X-ray pulses. The $\mathrm{MHz}$ data acquisition rate by the pulse trains will not be affected, however, and the time delays can be swiftly collected.

\section{Future experiments}

The results of this experiment at the SPB/SFX instrument of the European XFEL demonstrate that TR-SFX experiments are feasible at high repetition rate X-ray lasers. High power optical laser sources are required that match the specific $\mathrm{X}$-ray repetition rates and that are tunable to the photon energies needed to initiate reactions in biological macromolecules. The methods implemented here are generally applicable to comprehensively investigate macromolecular reactions within a dedicated experimental time, including built-in control measurements. The increased data rate at the EuXFEL may, in the future, support the collection of TR-SFX data within very limited experimental time (Supplementary Table. 6), assuming high uptime of all the necessary experimental apparatus. This opens the door to the deployment of enhanced analysis methods to extract macromolecular structures and their dynamics in the crystalline ensemble[39]. This has been achieved on slower timescales that were covered by dozens[25] of TRX datasets collected over multiple days, and often weeks, of beamtime at synchrotrons $[17,25,40]$. Close inspection and kinetic interpretation of the TR-SFX data allow an intimate view of mechanical aspects of signal transduction[41] and catalysis[42, 43], with specific atomic displacements linked to specific protein function. The close relationship between structural dynamics and function established in this way provides new avenues for the control[26, 44, 45] and understanding of biological function, which then also paves the way to a deeper understanding of the mechanism of biomacromolecular reactions in biomedically and biologically significant macromolecules.

\section{Methods}


A step-by-step protocol for a successful TR-SFX experiment with PYP at the EuXFEL is available on the Protocol Exchange[46]. This protocol can be readily modified for other (photoreactive) biological macromolecules.

\section{Sample preparation}

PYP was overexpressed and purified as reported[4, 18]. PYP microcrystals were grown with the stir method using $3.3 \mathrm{~mol}^{-1}$ malonate (pH 7) as precipitant[4]. PYP was concentrated to $100 \mathrm{mg} \mathrm{ml}^{-1}$, and $4 \mathrm{~mol}^{-1}$ Na-malonate, $\mathrm{pH} \mathrm{7,} \mathrm{was}$ added at once to a final concentration of $3.3 \mathrm{~mol} \mathrm{l}^{-1}$ under vigorous stirring. The suspension $(20 \mathrm{ml})$ was stirred in a closed glass vial for $8 \mathrm{~h}$ and allowed to rest for an additional $24 \mathrm{~h}$ at room temperature. This method works equally well with smaller $\sim 2 \mathrm{ml}$ and larger $\sim 20 \mathrm{ml}$ volumes. Stirring is necessary to prevent the growth of crystals to sizes larger than $10 \mu \mathrm{m}$. The slurry was spun at $8,000 \mathrm{~g}$ for $10 \mathrm{~min}$. The microcrystals swim up. The clear solution below the microcrystals was removed and replenished by $2.8 \mathrm{moll}^{-1} \mathrm{Na}$-malonate, $\mathrm{pH}$ 7. This resulted in a suspension containing about $10^{9}$ to $10^{10}$ crystals per ml, with most crystals being about $5 \mu \mathrm{m}$, as observed under a microscope with $\times 400$ magnification using a Neubauer cell counting chamber. The dense PYP microcrystalline slurry was filtered twice by handpressing the slurry (placed in a $10 \mathrm{ml}$ syringe) through a $10-\mu \mathrm{m}$ stainless steel filter. The slurry was injected without further filtering into the vacuum chamber at the SPB/SFX imaging and serial crystallography instrument [27, 47] using a GDVN[38] with $75 \mu \mathrm{m}$ inner diameter (Supplementary Fig. 1). Smaller nozzle diameters led to clogging and reduced flow rate due to pressure limitations.

\section{Injection and alignment}

With the $75 \mu \mathrm{m}$ inner diameter nozzles, we attempted to maximize slurry flow and gas pressure to produce a fast jet that may be able to cope with the enormous $\mathrm{X}$-ray pulse rates. We measured the jet diameter to be about $5 \mu \mathrm{m}$, with a flow rate of $35 \mu \mathrm{min}^{-1}$ under identical conditions to those used for the experiment (Supplementary Table. 1). This translates into a jet speed of approximately $30 \mathrm{~m}$ $\mathrm{s}^{-1}$. To avoid clogging, for some time delays, we added an immiscible fluorinated oil mixture (perfluorodecalin and $1 H, 1 H, 2 H, 2 H$-perfluoro-1-octanol in a 10:1 ratio) through a T-junction (Supplementary Fig. 1) located upstream of the injector nozzle rod at various flow rate ratios (Supplementary Table 1). The 
GDVN tip surface, as well as the capillaries to the GDVN, was treated with Novec 1720 (ref. [48]) to stabilize the simultaneous injection with oil and avoid growth of stalactite-like debris on the nozzle, which originates from the jet explosion after X-ray exposure. With this treatment, the oil can form a lubricant layer between the walls and the aqueous jet, which minimizes interaction and sticking of crystals and effectively avoiding clogging. As observed earlier[11], exposure to the intense X-ray pulses resulted in gaps in the jet caused by Coulomb explosion. The PYP photocycle was initiated in the microcrystals with $240 \mathrm{fs}$ laser pulses of wavelength $420 \mathrm{~nm}$, which is $30 \mathrm{~nm}$ on the blue side of the PYP absorption maximum (Supplementary Fig. 4b). As established earlier by ultrafast spectroscopy on PYP crystals[49], laser excitation (into the absorption maximum) with $1 \mathrm{~mJ} \mathrm{~mm}^{-2}$ is essentially free of nonlinear effects. This dispels concerns voiced recently about other systems[50]. Since the absorption at 420 $\mathrm{nm}$ is only $60 \%$ of that of the absorption maximum (Supplementary Fig. $4 \mathrm{~b}$ ), we used, accordingly, $1.6 \mathrm{~mJ} \mathrm{~mm}^{-2}$ laser fluence in a $42 \mu \mathrm{m}$ (FWHM) laser spot. Imaging the gaps in the jet[11] provides a convenient method to align the laser with the X-rays by centering the laser focal spot in the gap. Temperature was not controlled. Laser warming (about $10^{\circ} \mathrm{C}$ )[25] and evaporative cooling effects (a few degrees) fortuitously compensate each other. Details of the experimental setup, which includes laser set-up, laser alignment, the timing and the determination of the temporal overlap of X-ray and laser pulses are given in the following sections.

AQ10

\section{Instrumentation}

Experiments were performed at the SPB/SFX instrument in March 2019 as a part of proposal 2166 using a similar configuration as that used in Wiedorn et al.[11] (Supplementary Fig. 1). The size of the mirror-focused focal spot[51] in the interaction region was estimated to be $2 \times 3 \mu \mathrm{m}^{2}$ FWHM diameter based on optical imaging of single shots using a $20 \mu \mathrm{m}$ thick Ce:YAG screen. The X-ray pulse energy was about $700 \mu \mathrm{J}$. Diffraction from the sample was measured using an AGIPD[28, 52] of 1 megapixel located 117.7-118.6 mm downstream of the sample interaction region, with the unused direct beam passing through a central hole in the detector to a beam stop further downstream (Supplementary Fig. 1). The resolution at the edge of the AGIPD was $1.8 \AA$, and 1.6- $\AA$ data were obtained by integrating Bragg reflections into the detector corner. Experiment 
control was provided by Karabo[53] and data acquisition was provided by dedicated technology to cope with the megahertz repetition rates[54].

\section{Laser set-up and timing}

Optical laser radiation, with wavelength $840 \mathrm{~nm}$ and $15 \mathrm{fs}$ pulse duration, was used to generate a $420 \mathrm{~nm}$ second harmonic using a $0.5 \mathrm{~mm}$ thick beta barium borate (BBO) crystal. Additionally, fused silica windows and lenses stretched the pulse to $250 \mathrm{fs}$ duration. The beam size at the sample position was measured by a scintillator and a simple in situ microscope, yielding a diameter of $42 \mu \mathrm{m}$ FWHM. The average laser energy was about $2.3 \mu \mathrm{J}$, which corresponds to $1.6 \mathrm{~mJ}$ $\mathrm{mm}^{-2}$ fluence at the sample. The optical laser timing was synchronized with a radiofrequency (RF) signal, and timing at the experiment was controlled by a phase shifter. The T0 position, when both optical and X-ray beams arrive simultaneously, was estimated by the spatial encoding method using a $\mathrm{SrTiO}_{3}$ crystal[55]. Both the inter-train timing and the intratrain timing were measured earlier in facility experiments. The train arrival time jitter was determined to be $\sim 300$ fs for the system in RF lock[55]. The intratrain jitter was measured to be significantly shorter than $1 \mathrm{ps}$, which is negligible in terms of the ps timing scheme employed here. In addition, we never observed more than 1 ps drift in our RF synchronization over a $12 \mathrm{~h}$ shift.

\section{Data processing}

Experiment progress was monitored online using OnDA[56] for serial crystallography. Diffraction images with Bragg reflections were found by Cheetah[57] (peakfinder8, $\operatorname{minSNR}=8, \min A D C=200, \operatorname{minPix}=1, \operatorname{minPeaks}=$ 25 ) using the calibration process described by Wiedorn et al.[11]. Careful masking of shadowed and unreliable regions of the detector was performed on a run-by-run basis. Independent masks were used for peakfinding to avoid false peaks, for example, due to ice formation. Indexing was performed using CrystFEL v.0.8.0 with peaks found by Cheetah using the indexing package XGANDALF. Detector geometry, especially the detector distance, was refined using the program Geoptimiser[58]. Merging and scaling of the Bragg peak intensities were performed using the partialator program from CrystFEL. To avoid the integration of noise for weakly scattering patterns, reflections were included up to $1.0 \mathrm{~nm}^{-1}$ above a conservative resolution estimate for each crystal (--push-res $=1.0$ ). Since PYP crystallizes in $P 6_{3}$, an indexing ambiguity[59] is 
present[4]. This was corrected by the ambigator module in CrystFEL. Figures of merit were calculated using compare_hkl $\left(R_{\text {split }}, C C_{1 / 2}, C C^{*}\right)$ and check_hkl (signal-to-noise ratio, multiplicity, completeness), both a part of CrystFEL. The intensities from all indexed patterns were scaled together irrespective of the delay setting. Individual time delays were separated, and their intensity merged. This separation has been achieved based on pulse identities in the train (see below and Supplementary Fig. 1), which are stored together with the diffraction patterns. The corresponding intensities were then merged to generate reference datasets, and datasets at different time delays, for each (Supplementary Tables 1-3).

\section{Pump-probe timing schemes}

$\mathrm{X}$-ray pulses arrive in pulse trains with, currently, up to $176 \mathrm{X}$-ray pulses with a $1.13 \mathrm{MHz}$ repetition rate within the train. Each train repeats with $10 \mathrm{~Hz}$ (Fig. 2a and Supplementary Fig. 1). The tunable, high-energy femtosecond laser system installed at the SPB/SFX instrument[24] is able to cope with the MHz pulse repetition rate. For the various experiments, two different pump-probe timing strategies were used. These are shown in Fig. 2. The laser was synchronized to $\mathrm{X}$-ray pulse 1 in each train, whose radio frequency signal is delivered by the EuXFEL control room to the instrument. The X-ray fluence in pulse 1 has been very low for this experiment, and spurious diffraction patterns produced by it were not used. In scheme 1, the X-ray pulse rate was1.1 MHz (Fig. 2b). The laser was activated $2.3 \mathrm{~ns}$ after pulse 1 . Accordingly, pulses 2, 3 and 4 probed the reaction after $0.89 \mu \mathrm{s}(887 \mathrm{~ns}), 1.78 \mu \mathrm{s}$ and $2.67 \mu \mathrm{s}$. This sequence repeats with laser activation after pulses $4,7,10$ and so on, interleaved with the three $\mu$ s time delays each. This results in a laser pulse repetition rate of $376 \mathrm{kHz}$, and $176 / 3 \cong$ 58 laser activations per pulse train. The effective laser excitation rate is therefore $580 \mathrm{~Hz}$. As demonstrated in Supplementary Figs. 2a and 3, both the X-ray repetition rate and the intratrain laser repetition rate are too large for the jet speeds achieved with the viscous PYP microcrystalline slurry. In scheme 2 the $\mathrm{X}$-ray pulse repetition rate in the train is reduced to $564 \mathrm{kHz}$ with 88 pulses per train (Fig. 2c). This time, the hit rate varies smoothly with the X-ray pulse energy across all X-ray pulses in the train (Supplementary Fig. 2b), no abrupt decay as observed for higher X-ray pulse rates (as in Supplementary Fig. 2a) was observed. The laser was synchronized again with respect to X-ray pulse 1 . This time, the synchronization was precisely adjusted, so that the pump-probe delay 
$\Delta t$ between the laser pulse and X-ray pulse 2 was on the picosecond timescale (Fig. 2c), which is necessary to collect data for the $10 \mathrm{ps}, 30 \mathrm{ps}$ and $80 \mathrm{ps}$ time delays. As shown for other XFELs, the XFEL-to-laser timing fluctuations are of the order of $300 \mathrm{fs}$ (ref. [60]), which is negligible on the ps timescale, and a timing tool[61, 62] was not required. The next laser pulse arrives after X-ray pulse 5 . The laser pulse repetition rate was therefore $141 \mathrm{kHz}$. Accordingly pulses $2,6,10$, etc. in the train probe a picosecond time delay, and three additional interleaving X-ray pulses probe time delays at $1.78 \mu \mathrm{s}, 3.56 \mu \mathrm{s}$ and $5.33 \mu \mathrm{s}$. This results in 22 pump-probe sequences with ps time delays per train, and 220 effective laser excitations per second. At the Spring-8 Angstrom Compact Laser and the LCLS, typical X-ray pulse rates are $30 \mathrm{~Hz}$ and $120 \mathrm{~Hz}$, respectively. Pump-probe sequences with interleaving dark data collection require $15 \mathrm{~Hz}$ or $60 \mathrm{~Hz}$ laser pulse rates. Compared with these machines, even with the small number of $88 \mathrm{X}$-ray pulses in the train in these early experiments, the effective laser repetition rate of scheme 2 is a factor of 3.5 to 15 times faster. In the future, more than one order of magnitude more X-ray pulses will be available per train, which speeds up data collection accordingly. It must be decided on a case-by-case basis whether MHz pulse rates can be used when low viscosity slurries with small crystals are available that allow narrow GDVN orifices and enable fast jet speeds, or repetition rates of both X-ray and laser pulses must be reduced, as in the case of PYP. Of the order of $675 \mathrm{X}$-ray pulses per train, with a $1.13 \mathrm{MHz}$ intratrain repetition rate, are planned to be available soon. A pump-probe data collection strategy shown in Fig. 2a that contains only one interleaved dark will be feasible. In this case 337 laser pulses per train result in the enormous effective laser excitation rate of 3,370 Hz. As the AGIPD measures up to 352 pulses per train, 3,520 patterns (out of the 6,750) can be stored per second. With a low $2 \%$ hit rate and a $50 \%$ indexing rate, as demonstrated in this paper, the approximately 25,000 indexed diffraction patterns that are required to detect low levels of population transfer can be collected in about $20 \mathrm{~min}$. This includes collection of the reference (dark) data. Protein consumption is about $10 \%$ of that expected at slower XFELs (Supplementary Table. 6), and should be between $20 \mathrm{mg}$ and $40 \mathrm{mg}$ per time delay, depending on the design of the experiment.

\section{Difference map calculation}

A reference (dark state) model $M_{\text {ref }}$ was refined by using the program REFMAC 
(ref. [63]) against structure factor (SF) amplitudes collected in the dark $\left|F_{\text {dark }}\right|$ without laser excitation. To check for spurious features at $\mu$ s delays, the pure dark data (Supplementary Table 2) were used as a reference. The dark3 data from the 30 ps time series display superior statistics (Supplementary Table 3), and served as reference for all ps time delays. Model structure factors were calculated from $M_{\text {ref }}$ with amplitude $\left|\mathrm{FC}_{\text {ref }}\right|$ and phase $\phi_{\text {ref }}$. The measured $\left|F_{\text {dark }}\right|$ were brought to the absolute scale by scaling them to $\left|\mathrm{FC}_{\text {ref }}\right|$ using the CCP4 program 'scaleit' (ref. [64]). The time-dependent SF amplitudes $\left|F_{\text {obs }}(t)\right|$ were then scaled to the $\left|F_{\text {dark }}\right|$ in a second run of 'scaleit'. As a result, both $\left|F_{\text {obs }}(t)\right|$ and $\left|F_{\text {dark }}\right|$ are on the absolute scale, and are scaled together. Difference structure factor (DSF) amplitudes were calculated as: $\mathrm{DSF}_{\text {obs }}=\left|F_{\text {obs }}(t)\right|-\left|F_{\text {dark }}\right|$. A weighting factor, $w$, for the DSFs was determined to reduce the influence of outliers[65]. The DSFs were combined with phases $\phi_{\text {ref }}$. From the weighted DSFs, a weighted DED map was calculated using the program ' $\mathrm{fft}$ ' from the CCP4 suite of programs[64]. Although the $\left|F_{\text {obs }}(t)\right|$ and the $\left|F_{\text {dark }}\right|$ are on the absolute scale, the difference map was, due to the difference Fourier approximation[66], only on half the absolute scale. The preserved absolute scale was necessary to estimate population transfer levels, as explained below. The DED maps were best contoured on the $3 \sigma /-3 \sigma$ levels (Supplementary Fig. 6)

\section{Refinement}

Meaningful negative features in the DED map were necessarily located on top of the reference model $M_{\text {ref }}$. However, contiguous, chemically sensible positive features in the DED map must be interpreted with a new structural model $\left(M_{\mathrm{TRX}}\right)$. To determine structures from DED maps, extrapolated, conventional electron density $\left(\mathrm{ED}_{\mathrm{ext}}\right)$ maps[14, 16] were used. For extrapolated structure factor $\left(\mathrm{SF}_{\text {ext }}\right)$ amplitudes a multiple of the DSFs were added to the $\left|\mathrm{FC}_{\text {ref }}\right|$ : $S F_{\text {ext }}=\left|F C_{\text {ref }}\right|+N \times D S F$ and combined with the reference state (dark) phases $\phi_{\text {ref }}$. Here the use of $\left|\mathrm{FC}_{\text {ref }}\right|$ derived from an accurately refined dark state model was preferred over the $\left|F_{\text {dark }}\right|$, as explained by Terwilliger and Berendsen[67]. From the phased $\mathrm{SF}_{\text {ext }}$, extrapolated electron density maps $\left(\mathrm{ED}_{\text {ext }}\right)$ were calculated with the CCP4 program ' $\mathrm{fft}$ '. A characteristic $N_{\text {ext }}$ was established when the electron density in the $\mathrm{ED}_{\text {ext }}$ at the positions with strong negative features in the DED maps just vanishes. When $N$ is too large, false-negative features will appear in the $\mathrm{ED}_{\text {ext }}$. This can be visualized by summing up negative values in the $\mathrm{ED}_{\text {ext }}$ within a volume that contains strong DED features in the 
DED maps. Supplementary Fig. 7 shows results for such a summation for all our TR-SFX time delays collected at the EuXFEL and for TRX data selected from the literature $[4,14,33]$. The $N_{\text {ext }}$ is marked with an arrow in Supplementary Fig. 7. The value of $N_{\mathrm{ext}}$ is approximately related to the PT: $P T \approx \frac{100}{N_{\mathrm{ext}}} \times 2(\%)$. The factor of two accounts for the difference Fourier approximation mentioned above. If the PT is small, $N_{\text {ext }}$ is large. A value of $N_{\text {ext }}=30$ is not uncommon in TRX, especially with fs excitation, since the primary yield of photoactivation can be quite small[34] and is further diminished by experimental circumstances. Once $N_{\text {ext }}$ had been established, structural models were determined from the resulting $\mathrm{ED}_{\text {ext }}$ maps. The $\mathrm{ED}_{\text {ext }}$ map was displayed in a molecular modeling program such as 'coot'[68]. The reference model can be used as an initial model for a refinement. When structural changes were small, the initial model was altered by directly refining it against the $\mathrm{ED}_{\text {ext }}$ map by a stepped real-space refinement in 'coot' with the torsional restraint switched off (default in 'coot'). For PYP, isomerization and structural changes were modeled automatically in this way, entirely without manual intervention. For other systems[69] structural changes can be modeled manually, analogous to conventional structure determination. A new structural model $M_{\mathrm{TRX}}$ was obtained this way. From the real-space refined $M_{\mathrm{TRX}}$ and $M_{\text {ref }}$ models, calculated $\mathrm{DSF}_{\text {calc }}$ can be determined, this time with amplitude and phases $\phi_{\Delta}$. When the $\mathrm{DED}_{\text {calc }}$ calculated from the phased difference structure factors is compared with the $\mathrm{DED}_{\text {obs }}$, prominent DED features should match (Supplementary Fig. 8). The $\phi_{\Delta}$ were combined with the measured $\mathrm{DSF}_{\mathrm{obs}}$ and phased extrapolated SFs (pSF) were obtained by adding the (now phased) $\mathrm{DSF}_{\text {obs }}$ to the $\mathrm{FC}_{\text {ref }}$ as vectors in the complex plane[31, 32]. The $M_{\mathrm{TRX}}$ was refined against the $|\mathrm{pSF}|$ using restrained reciprocal space refinement, using, for example, 'refmac'[63]. Typically, $R$-factors were acceptable and did not deviate much from those of refinements against conventional X-ray data. Structural models and ED maps are shown in Supplementary Fig. 3. Refinement statistics are shown in Supplementary Tables 4 and 5. Selected model parameters are listed in Table 1 for the 3 ps to 100 ps time range.

AQ11

\section{Displacements and difference distance matrices}

Structural differences were analyzed by calculating the root mean square displacements of like $\mathrm{C}_{\alpha}$ atoms in the $M_{41-71}$ moiety (Supplementary Fig. 4a) 
between the structures determined at the various time delays and the reference structure. The root mean square displacements values in Supplementary Fig. 4c were fit by an empirical function consisting of an exponential term, a linear term and a strongly damped cosine function, which includes a phase shift:

$$
\mathrm{RMSD}_{\mathrm{fit}}=A_{0}\left(1-\mathrm{e}^{-\frac{1}{\tau} t}\right)+b \cdot t+A_{1} \cos \left(\frac{2 \pi}{T} t+\varphi\right) \mathrm{e}^{-\beta t}
$$

\section{AQ12}

AQ13

The fit values were $A_{0}=0.181 \AA, \tau=1.4 \mathrm{ps}, b=4.5 \times 10^{-3} \AA \mathrm{ps}^{-1}, A_{1}=0.038 \AA$, $T=50 \mathrm{ps}, \varphi=257^{\circ}, \beta=1 / 50 \mathrm{ps}^{-1}$. Some of the fit values are not unique. For example, $T$ in the cosine function can vary largely because only a few time delays are available across the $100 \mathrm{ps}$ timescale. $T$ was selected so that the decrease of the root mean square displacements at 30 ps was modeled correctly, and the damping constant $\beta$ was selected so that the oscillation vanishes quickly. To show more global displacements, difference distance matrices (Supplementary Fig. 5a-d) were calculated[70] using the Fortran code of the DDMP program from the Center for Structural Biology at Yale University. The calculations include residues 42-92 and use the 3 ps structure as a reference (note: if the dark structure were to be used as a reference, small structural changes in the time window from 3 ps to 100 ps would not be observable). With these matrices one can also visually identify the decline in the magnitude of the difference distances between $10 \mathrm{ps}$ and $30 \mathrm{ps}$, and their increase at $100 \mathrm{ps}$, in particular in the $M_{41-71}$ region (green bar), and then at 100 ps also more globally in the $\mathrm{H}_{74-88}$ region (also depicted in Supplementary Fig. 4a).

\section{Reporting Summary}

Further information on research design is available in the Nature Research Reporting Summary linked to this article.

\section{Online content}

Any methods, additional references, Nature Research reporting summaries, source data, extended data, supplementary information, acknowledgements, peer review information; details of author contributions and competing interests; and 
statements of data and code availability are available at https://doi.org/10.1038 /s41592-019-0628-z.

Supplementary information

Supplementary information is available for this paper at https://doi.org/10.1038 /s41592-019-0628-z.

Peer review information Arunima Singh and Allison Doerr were the primary editors on this article and managed its editorial process and peer review in collaboration with the rest of the editorial team

Publisher's note Springer Nature remains neutral with regard to jurisdictional claims in published maps and institutional affiliations.

\section{Acknowledgements}

We acknowledge European XFEL in Schenefeld, Germany, for provision of X-ray free-electron laser beamtime at Scientific Instrument SPB/SFX and thank the instrument group and facility staff for their assistance. This work was supported by NSF Science and Technology Centers (grant no. NSF-1231306; Biology with X-ray Lasers) to H.N.C., P.F., A.O., A.R., P.S. and M.S.). CFEL (H.N.C.) is supported by the Gottfried Wilhelm Leibniz Program of the DFG; the project 'X-probe' funded by the European Union's 2020 Research and Innovation Program under the Marie Sklodowska-Curie grant agreement (no. 637295); the European Research Council, 'Frontiers in Attosecond X-ray Science: Imaging and Spectroscopy (AXSIS)' (no. ERC-2013-SyG 609920, together with P.F.); and the Human Frontiers Science Program grant (no. RGP0010 2017). P.F. and A.R. acknowledge the support of funding from the Biodesign Center for Applied Structural Discovery at Arizona State University and NSF award (no. 1565180). Funding from the National Institutes of Health (grant nos. R01GM095583 to P.F. and R01GM117342 to M.F.) is also acknowledged.

AQ14

Author contributions

S.P., I.P. and M.S. expressed, purified and crystallized the protein. R.B., T.S., J.B., V.B., M.E., G.G., M.J., Y.K., H.K., A.K., R.L., L.M., T.M., G.P., M.R., A.S., J.S.-D. and A.P.M. operated the SPB/SFX instrument. S.L., J.K., R.S. and H.N.C. provided injector nozzles. J.C.V., C.K., M.H., M.H.A., J.K., F.H.M.K., 
S.L., V.M., D.M., R.S. and A.T. collected the data. S.P., I.P., O.Y., V.M., T.A.W., Y.G., A.O., P.S., A.T. and A.B. processed the data. S.P., I.P., P.S., A.O. and M.S. analyzed the data. C.K., M.H.A., R.F. and P.F. logged the experiment. J.C.V., A.E., D.D., D.K. and A.R. conceived and operated the oil co-flow. R.B., T.S., M.F., H.N.C., A.R., A.B., P.F., A.P.M. and M.S. designed the experiment. S.P., S.B., A.B., P.F., A.P.M. and M.S. wrote the manuscript with input from all other authors.

AQ15

\section{Data availability}

Data has been deposited with the Coherent X-ray Imaging Data Bank[71, 72, 73] with CXIDB ID 100. This includes: stream files for all data and for data separated into each time delay, MTZ and PDB files for all time delays, including the dark/reference structures. We have deposited data (mtz-files and structures) for the $10 \mathrm{ps}, 30 \mathrm{ps}$ and $80 \mathrm{ps}$ time delays, as well as the dark3 (30 ps) and pure dark reference structures, with the Protein Data Bank, with deposition codes 6P4I, 6P5D, 6P5E, 6P5G and 6P5F, respectively.

AQ16

AQ17

AQ18

\section{Code availability}

Linux scripts and Fortran source codes for the calculation of weighted difference maps, extrapolated electron density maps and the integration of negative densities within a spherical volume are included in a demonstration, which is available online as Supplementary Data.

Competing interests The authors declare no competing interests. AQ19

\section{Integrated supplementary information}

\section{Supplementary Fig. 1}

Setup of a MHz TR-SFX experiment at the EuXFEL (modified from Wiedorn et al.[11]).

$\mathrm{X}$-ray pulses arrive in $1.13 \mathrm{MHz}$ bursts which repeat every $100 \mathrm{~ms}$. There are 176 $\mathrm{X}$-ray pulses in the burst. The KB-mirror system focuses the X-ray beam to a 2-3 
$\mu \mathrm{m}$ focal spot. The fs-laser delivers $376 \mathrm{kHz}$ pulses $(\lambda=420 \mathrm{~nm}$, blue) synchronized to the X-ray pulses. The laser focus is $42 \mu \mathrm{m} \varnothing$ in the X-ray interaction region (dotted circle). The microcrystals are mixed with fluorinated oil and injected by a GDVN. The jet produced by the GDVN, the laser beam as well as the X-ray pulses precisely intersect. The time-resolved diffraction patterns are collected by the AGIPD. Diffraction patterns with common time-delays were separated based on the pulse ID (see also Fig. 2b) and combined to datasets.

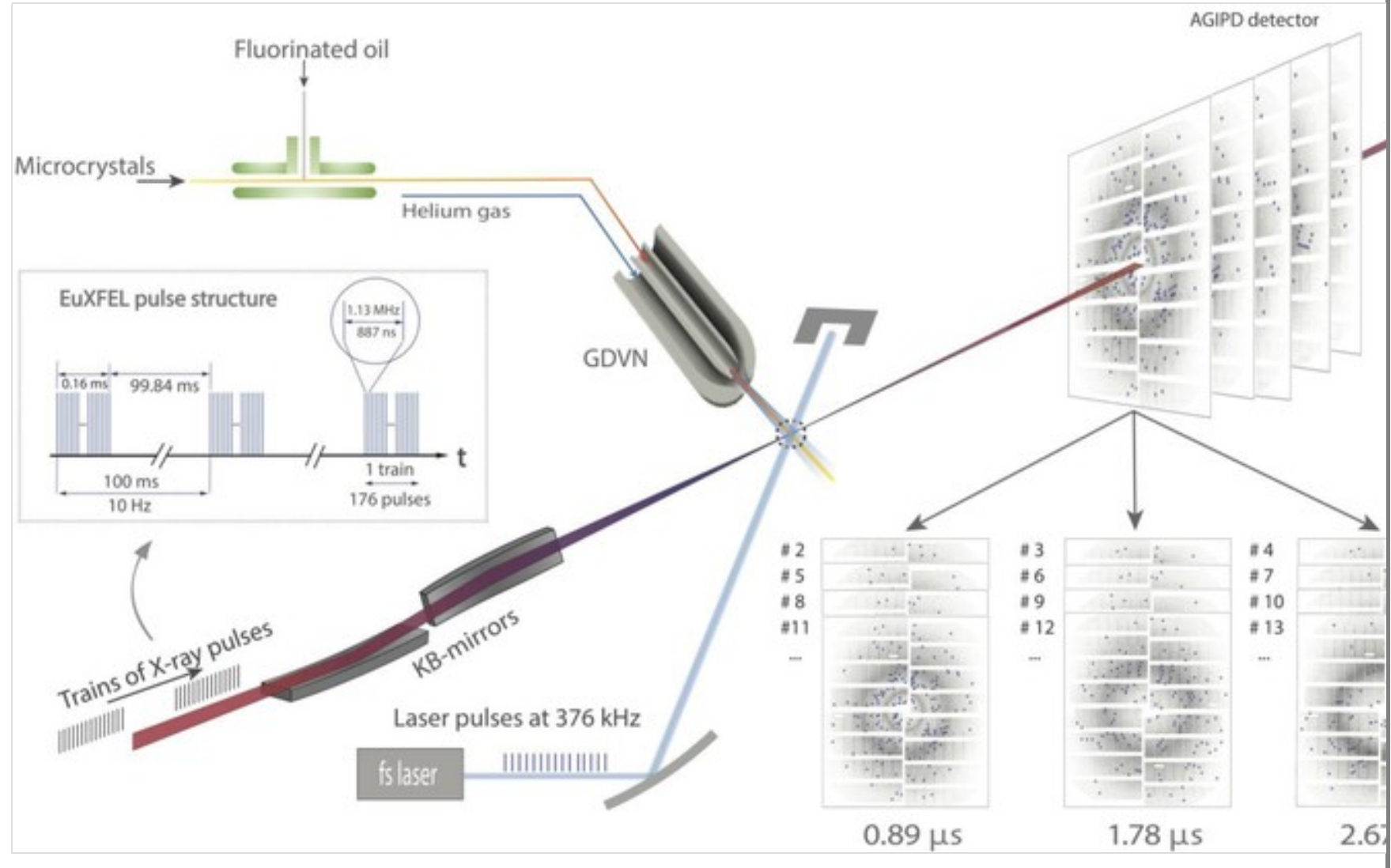

\section{Supplementary Fig. 2}

Hit and indexing rates.

a, Hit rates (red) and indexing rates (black) with $1.13 \mathrm{MHz}$ X-ray pulse repetition rate. Note, the strong drop of the hit-rate after the first pulse from $2 \%$ to $1 \%$. 472,528 total patterns, 41,559 hits and 24,815 indexed patterns were separated on the basis of pulse IDs. From these, hit rates and indexing rates were calculated. b, Hit rates (red) and indexing rates (black) with $564 \mathrm{kHz}$ X-ray pulse repetition. The overall hit rate is about 2\%. 52,495,158 total patterns, 304,673 hits and 142,948 indexed patterns were separated on the basis of pulse IDs from which hit rates and 
indexing rates were calculated. Blue solid line in $\mathbf{a}$ and $\mathbf{b}, \mathrm{X}$-ray pulse energy (on arbitrary scale). The indexing rate varies only slightly and is about $40 \%-60 \%$.
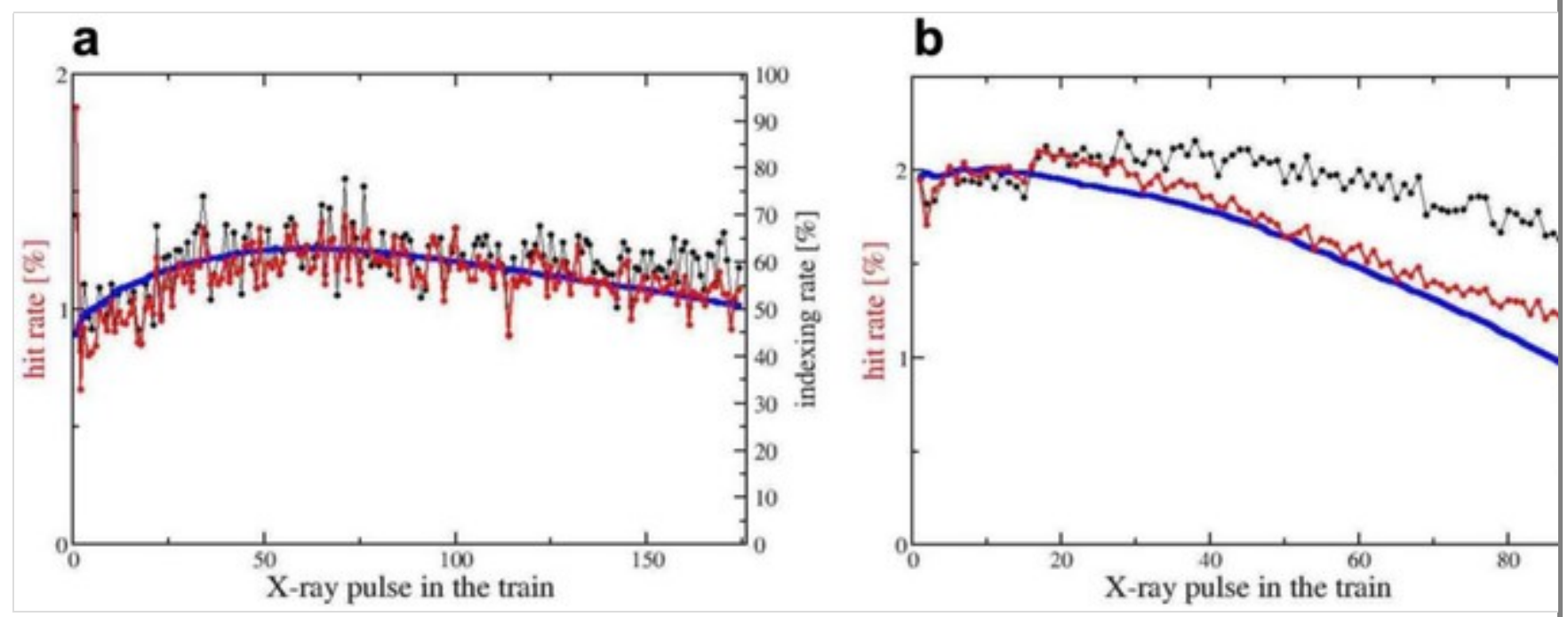

\section{Supplementary Fig. 3}

Extrapolated electron density maps (1.5 $\sigma$ contour level).

a, 3 ps at LCLS (Pande et al.[14]). b - c, 10 ps, 30 ps and 80 ps at EuXFEL. e, 100 ps at APS (Jung et al. [33]). The extrapolated maps were calculated from 13,722, $13,142,13,014,12,889$ and 13,214 extrapolated structure factors for the 3 ps to 100 ps time delays, respectively. 


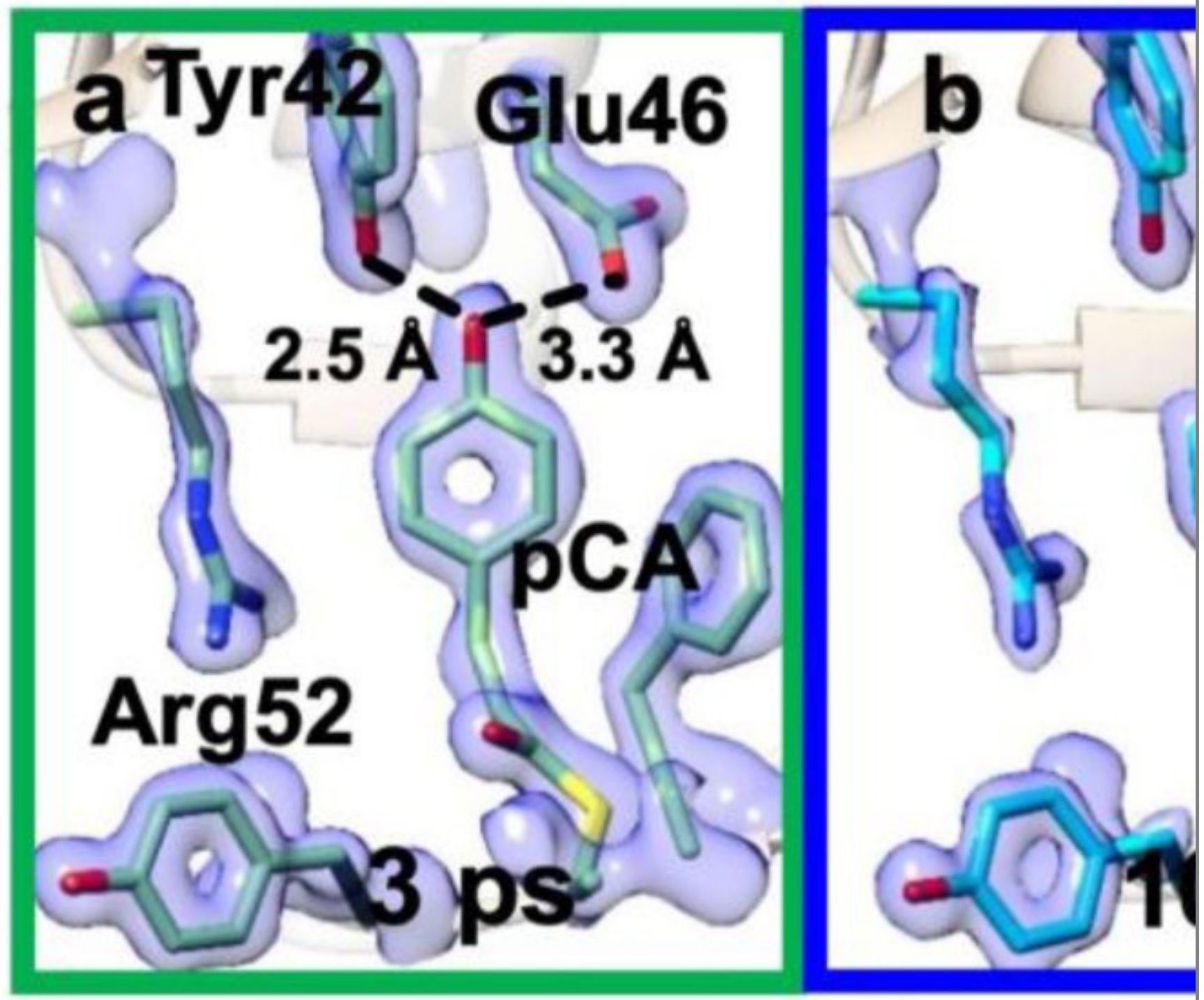

\section{Supplementary Fig. 4}

\section{Excitation and ultrafast displacements in PYP.}

a, Structure of PYP. Some important residues in the chromophore (pCA) binding pocket are marked. The $\mathrm{M}_{41-71}$ moiety (residues 41 to 71 ) is marked in red. Helix $\mathrm{H}_{74-88}$ is marked. b, Dark state spectra of PYP. Black: measured in solution, red: in the crystal. The wavelength at the absorption maximum is marked. Excitation has been achieved with 240 fs laser pulses with $\lambda=420 \mathrm{~nm}$. c, Solid spheres: root mean square displacements of $31 \mathrm{C}_{\alpha}$ atoms in $\mathrm{M}_{41-71}$ relative to the dark (reference) structure, red spheres: from data measured at EuXFEL. Dashed line: fit by a function consisting of an exponential, a strongly damped, phase shifted cosine 
function and a straight line as outlined in the text.
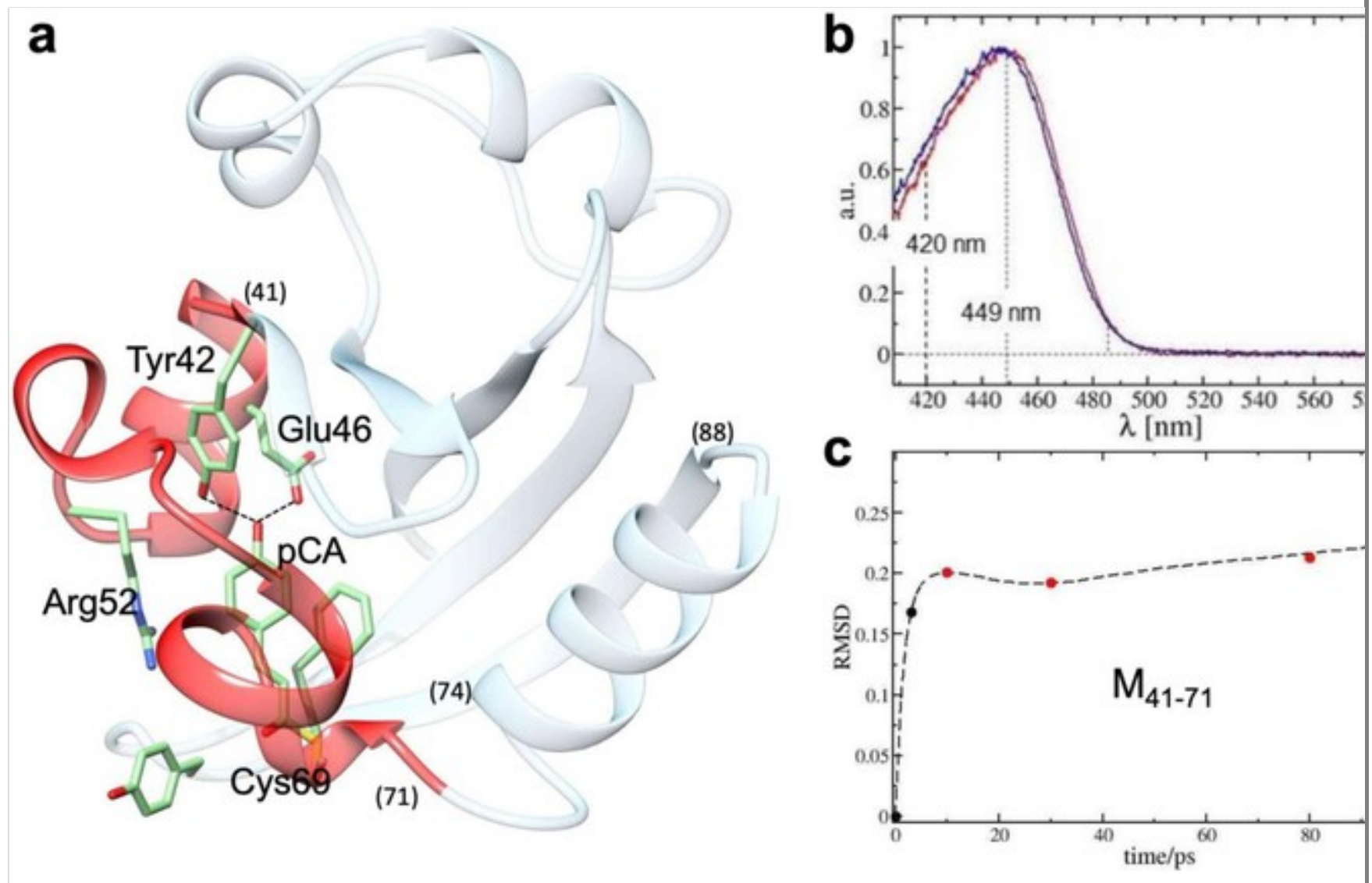

\section{Supplementary Fig. 5}

Difference distance matrices evaluated for $\mathrm{C}_{\alpha}$ atoms of residues 42 to 93 .

The green line denotes the $\mathrm{M}_{41-71}$ moiety. The scale on top is in $\AA$. a - d, Difference distance matrices derived from structures at $10 \mathrm{ps}, 30 \mathrm{ps}, 80 \mathrm{ps}$ and 100 ps relative to that at $3 \mathrm{ps}$, respectively. Difference distances are also shown for helix $\mathrm{H}_{74-88}$. 
$-0.500 \bigcirc-0.400 \bigcirc-0.300 \bigcirc-0.200 \bigcirc-0.100-0.000$

0.100

0.200

$0.300 \bigcirc 0.400$

0.500

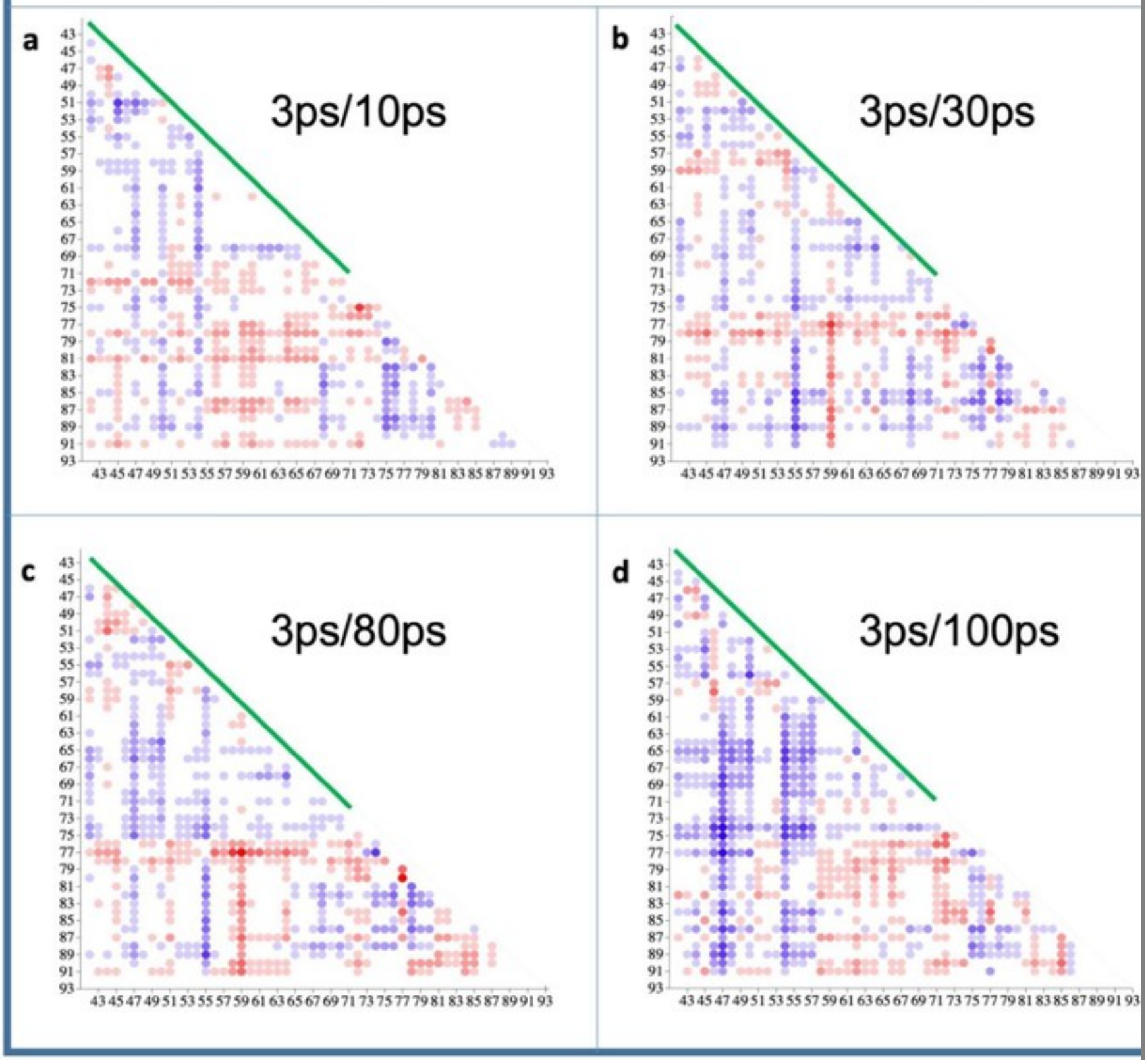

\section{Supplementary Fig. 6}

Signal levels in the DED map at the 30 ps delay.

The DED map at $30 \mathrm{ps}$ is overlaid on the entire PYP and contoured from $+/-2 \sigma$ to $+/-4 \sigma$ in steps of $0.5 \sigma$. Red: negative DED, green: positive DED. The $3 \sigma$ level, $\mathbf{c}$, is the best compromise to distinguish the signal, for example on the pCA chromophore, from spurious noise features distributed within the protein volume. 


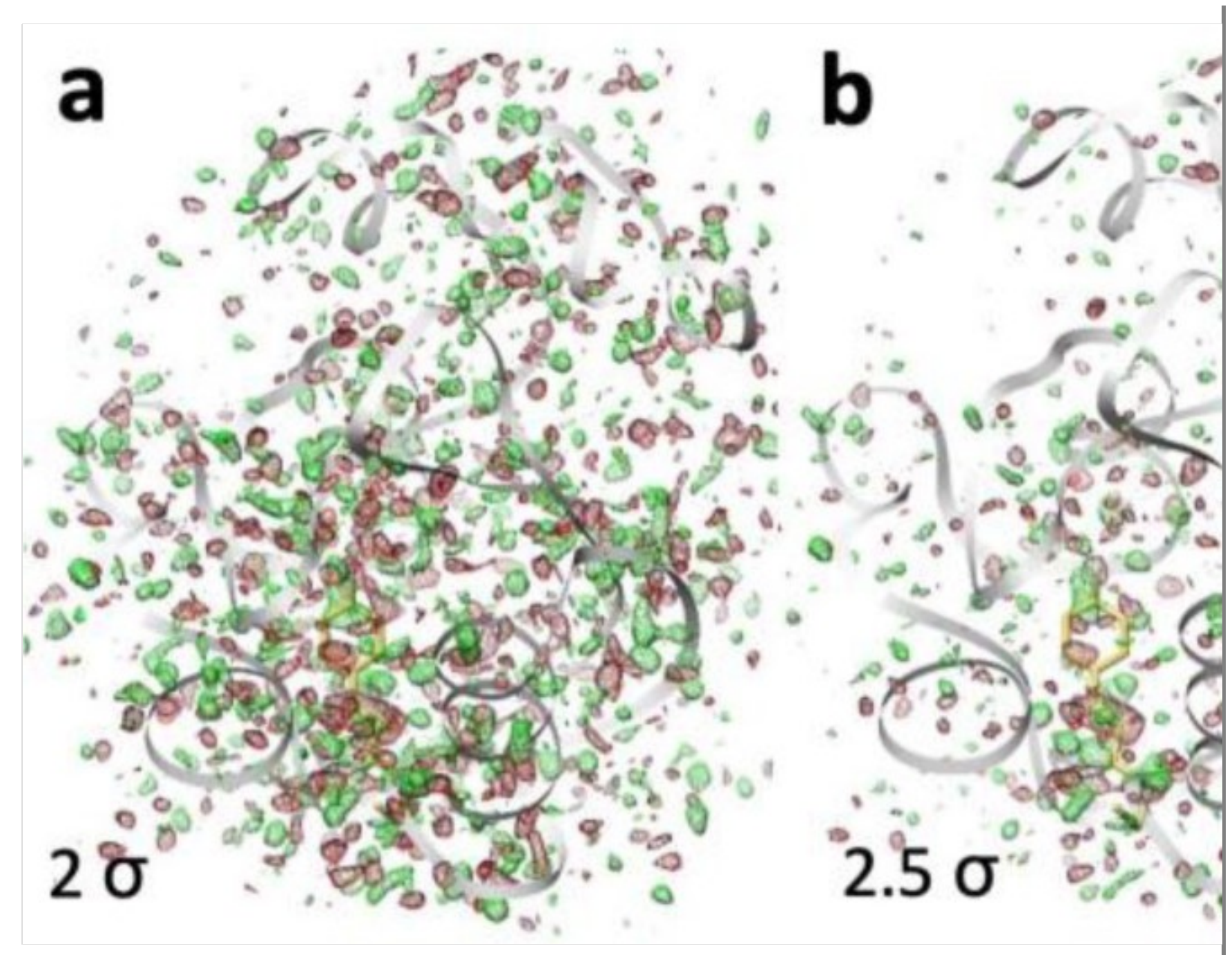

\section{Supplementary Fig. 7}

Method to determine the factor $\mathrm{N}$ and the population transfer (PT).

The factor $\mathrm{N}$ has been determined to calculate extrapolated, conventional maps from data collected at various X-ray sources. Black spheres: summed absolute negative DED in a sphere of $\mathrm{R}=4 \AA$ centered on the PCA chromophore double bond. Red dotted lines: the more horizontal line follows the initial slope of the data; the second line delineates the constant incline with larger Ns. The $\mathrm{N}_{\text {ext }}$ (in brackets) can be estimated from the intersection of the two lines. a, 3ps data from CXI at LCLS collected with fs laser excitation in the absorption maximum (Pande et al. [14]). Factor $\mathrm{N}=16, \mathrm{PT}=12.5 \%$, insert: $1 \mu$ s data collected with ns laser excitation. $\mathrm{N}=4$, and $\mathrm{PT}=50 \%$ (Tenboer et al.[4]). $\mathbf{b}$, $\mathbf{c}$, and $\mathbf{d}$, Factors $\mathrm{N}$ for the $10 \mathrm{ps}, 30 \mathrm{ps}$, and $80 \mathrm{ps}$ data collected at the EXFEL with fs laser excitation outside the absorption maximum. PT is about $7 \%$ throughout. Insert in d, 100 ps data 
collected at APS (about 6\% PT, Jung et al.[33]). 13,214, 13,542, 13,722, 13,142, 13,014 and 12,889 observed difference amplitudes are used to determine extrapolated maps for the 100ps, $1 \mu \mathrm{s}, 3 \mathrm{ps}, 10 \mathrm{ps}, 30 \mathrm{ps}$ and $80 \mathrm{ps}$ time delays, respectively.

a
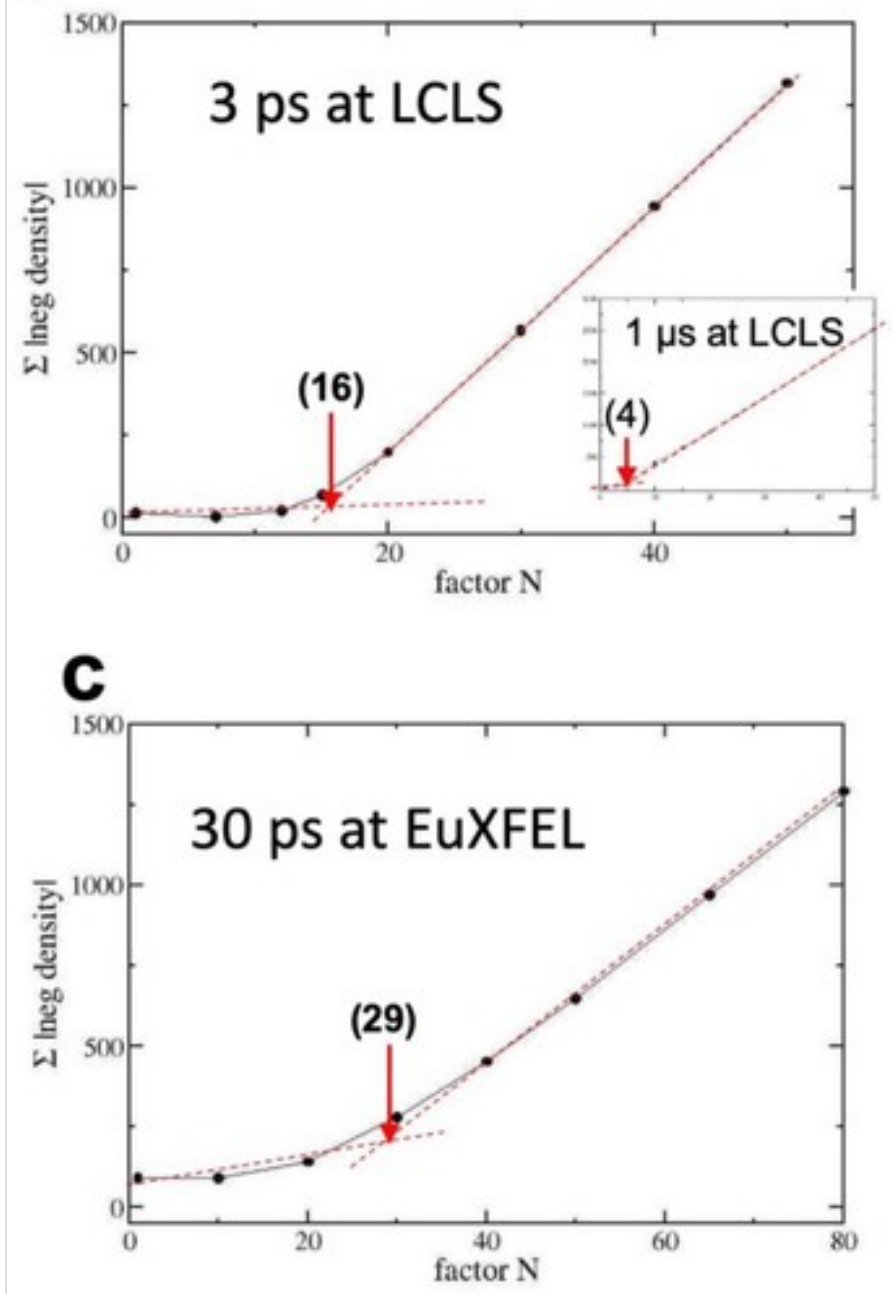
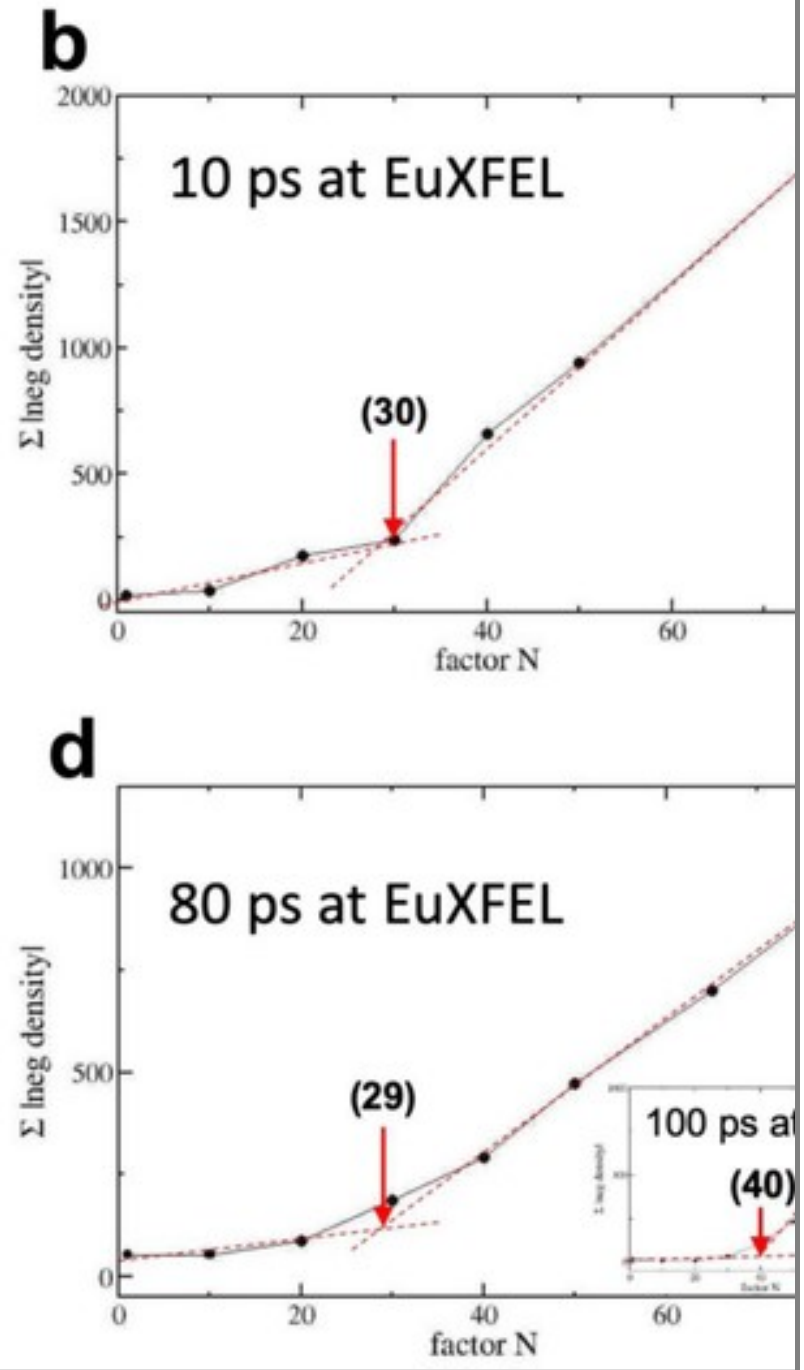

\section{Supplementary Fig. 8}

Observed and calculated difference electron densities (DED) near the pCA chromophore.

Left panels: observed difference electron density (blue: $3 \sigma$, red: $-3 \sigma$ contour levels). Right panels: calculated difference electron density (blue: $4 \sigma$, red: $-4 \sigma$ contour levels). Yellow model: structure of the dark (reference) state; blue model: structure at a particular time delay. a, $10 \mathrm{ps} ; \mathbf{b}, 30 \mathrm{ps}, \mathbf{c}, 80 \mathrm{ps}$. In panel b pairwise difference density features are marked with $\alpha$ (negative) and $\beta$ (positive). The 
feature $\gamma$ shows the signal caused by the Cys-69 sulfur. The marked DED features can be readily detected at the other time delays. 13,142, 13,014 and 12,889 difference amplitudes were used to calculate the observed DED maps for $\mathbf{a}, \mathbf{b}$ and c, respectively.
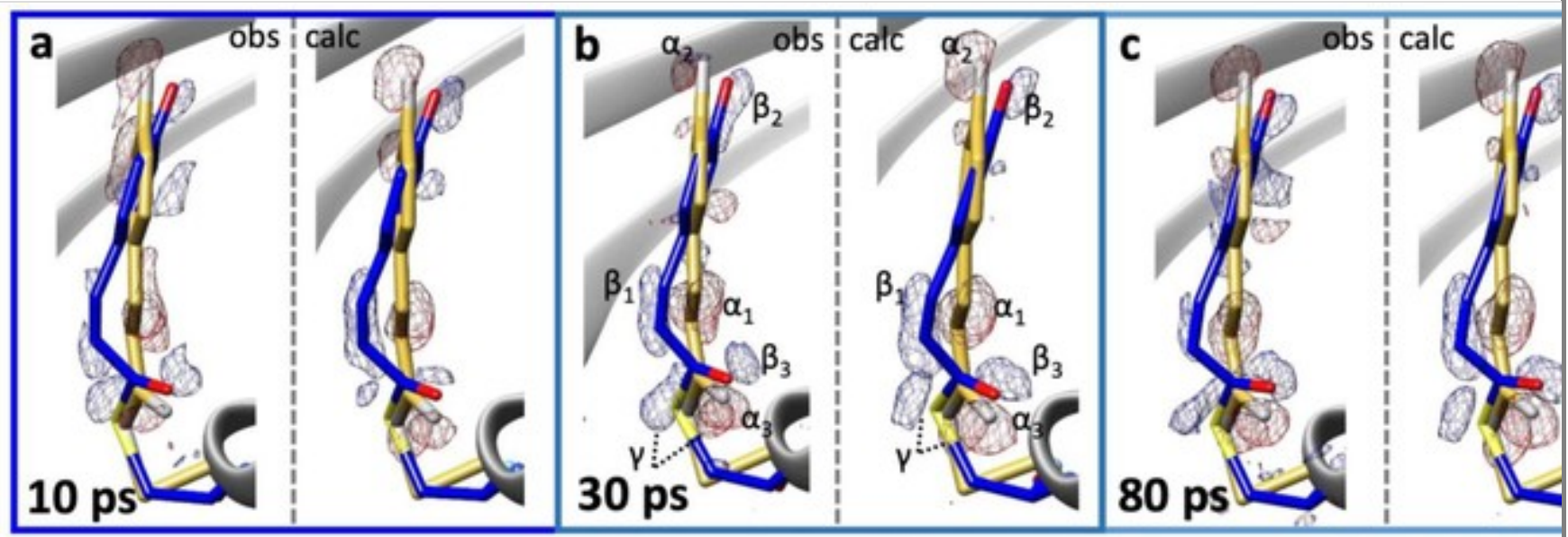

\section{Supplementary information}

\section{Supplementary Information}

Reporting Summary

Supplementary Figs. 1-8 and Tables 1-7.

\section{Supplementary Software}

demo.tar.zip. Compressed repository containing a demonstration and software for difference map calculation and structure determination. After the TR-SFX experiment datasets of reference (dark) and time-dependent intensities are available. This demonstration guides through the processes of difference map calculation and structure determination from extrapolated electron density maps.

\section{References}

1. Moffat, K. Time-resolved biochemical crystallography: a mechanistic perspective. Chem. Rev. 101, 1569-1581 (2001). 
2. Schmidt, M. Time-resolved macromolecular crystallography at modern X-ray sources. Methods Mol. Biol. 1607, 273-294 (2017).

3. Aquila, A. et al. Time-resolved protein nanocrystallography using an X-ray free-electron laser. Opt. Express 20, 2706-2716 (2012).

4. Tenboer, J. et al. Time-resolved serial crystallography captures highresolution intermediates of photoactive yellow protein. Science 346, 1242-1246 (2014).

5. Chapman, H. N. et al. Femtosecond X-ray protein nanocrystallography. Nature 470, 73-77 (2011).

6. Boutet, S. et al. High-resolution protein structure determination by serial femtosecond crystallography. Science 337, 362-364 (2012).

7. Lomb, L. et al. Radiation damage in protein serial femtosecond crystallography using an X-ray free-electron laser. Phys. Rev. B 84, 214111 (2011).

8. Nass, K. et al. Indications of radiation damage in ferredoxin microcrystals using high-intensity X-FEL beams. J. Synchrotron Radiat. 22, 225-238 (2015).

9. Suga, $\mathrm{M}$. et al. Light-induced structural changes and the site of $\mathrm{O}=\mathrm{O}$ bond formation in PSII caught by XFEL. Nature 543, 131-135 (2017).

10. Chreifi, G. et al. Crystal structure of the pristine peroxidase ferryl center and its relevance to proton-coupled electron transfer. Proc. Natl Acad. Sci. USA 113, 1226-1231 (2016).

11. Wiedorn, M. O. et al. Megahertz serial crystallography. Nat. Commun. 9, 4025 (2018).

12. Grunbein, M. L. et al. Megahertz data collection from protein microcrystals at an X-ray free-electron laser. Nat. Commun. 9, 3487 (2018). AQ20 
13. Barends, T. R. et al. Direct observation of ultrafast collective motions in CO myoglobin upon ligand dissociation. Science 350, 445-450 (2015).

14. Pande, K. et al. Femtosecond structural dynamics drives the trans/cis isomerization in photoactive yellow protein. Science 352, 725-729 (2016).

15. Meyer, T. E., Yakali, E., Cusanovich, M. A. \& Tollin, G. Properties of a water-soluble, yellow protein isolated from a halophilic phototrophic bacterium that has photochemical activity analogous to sensory rhodopsin. Biochemistry 26, 418-423 (1987).

16. Genick, U. K. et al. Structure of a protein photocycle intermediate by millisecond time-resolved crystallography. Science 275, 1471-1475 (1997).

17. Ihee, H. et al. Visualizing reaction pathways in photoactive yellow protein from nanoseconds to seconds. Proc. Natl Acad. Sci. USA 102, 7145-7150 (2005).

18. Kort, R. et al. Evidence for trans-cis isomerization of the $p$-coumaric acid chromophore as the photochemical basis of the photocycle of photoactive yellow protein. FEBS Lett. 382, 73-78 (1996).

19. Polli, D. et al. Conical intersection dynamics of the primary photoisomerization event in vision. Nature 467, 440-443 (2010).

20. Mathes, T. et al. Femto- to microsecond photodynamics of an unusual bacteriophytochrome. J. Phys. Chem. Lett. 6, 5 (2014).

21. Ali, A. M. et al. Optogenetic inhibitor of the transcription factor CREB. Chem. Biol. 22, 1531-1539 (2015).

22. Schotte, $F$. et al. Watching a signaling protein function in real time via 100-ps time-resolved Laue crystallography. Proc. Natl Acad. Sci. USA 109, 19256-19261 (2012).

23. Creelman, M., Kumauchi, M., Hoff, W. D. \& Mathies, R. A.

Chromophore dynamics in the PYP photocycle from femtosecond stimulated 
Raman spectroscopy. J. Phys. Chem. B 118, 659-667 (2014).

24. Palmer, G. et al. Pump-probe laser system at the FXE and SPB/SFX instruments of the European X-ray free-electron laser facility. J. Synchrotron Radiat. 26, 328-332 (2019).

25. Schmidt, M. et al. Protein energy landscapes determined by fivedimensional crystallography. Acta Crystallogr. D 69, 2534-2542 (2013).

26. Prokhorenko, V. I. et al. Coherent control of retinal isomerization in bacteriorhodopsin. Science 313, 1257-1261 (2006).

27. Mancuso, A. P. et al. The single particles, clusters and biomolecules and serial femtosecond crystallography instrument of the European XFEL: initial installation. J. Synchrotron Radiat. 26, 660-676 (2019).

28. Allahgholi, A. et al. The adaptive gain integrating pixel detector at the European XFEL. J. Synchrotron Radiat. 26, 74-82 (2019).

29. Stan, C. A. et al. Liquid explosions induced by X-ray laser pulses. Nat. Phys. 12, 966-971 (2016).

30. Tripathi, S., Srajer, V., Purwar, N., Henning, R. \& Schmidt, M. pH dependence of the photoactive yellow protein photocycle investigated by time-resolved crystallography. Biophys. J. 102, 325-332 (2012).

31. Schmidt, M. in Ultrashort Laser Pulses in Medicine and Biology (eds Braun, M. et al.) 201-241 (Springer, 2008).

32. Schmidt, M. Time-resolved macromolecular crystallography at pulsed X-ray sources. Int. J. Mol. Sci. 20, 1401 (2019).

AQ21

33. Jung, Y. O. et al. Volume-conserving trans-cis isomerization pathways in photoactive yellow protein visualized by picosecond X-ray crystallography. Nat. Chem. 5, 212-220 (2013).

34. Hutchison, C. D. M. \& van Thor, J. J. Populations and coherence in 
femtosecond time resolved X-ray crystallography of the photoactive yellow protein. Int, Rev. Phys. Chem. 36, 117-143 (2017).

35. Groenhof, G. et al. Photoactivation of the photoactive yellow protein: why photon absorption triggers a trans-to-cis isomerization of the chromophore in the protein. J. Am. Chem. Soc. 126, 4228-4233 (2004).

36. Markovitch, O. \& Agmon, N. Structure and energetics of the hydronium hydration shells. J. Phys. Chem. A 111, 2253-2256 (2007).

37. Levantino, M. et al. Ultrafast myoglobin structural dynamics observed with an X-ray free-electron laser. Nat. Commun. 6, 6772 (2015).

38. DePonte, D. P. et al. Gas dynamic virtual nozzle for generation of microscopic droplet streams. J. Phys. D 41, 195505 (2008).

AQ22

39. Schmidt, M., Rajagopal, S., Ren, Z. \& Moffat, K. Application of singular value decomposition to the analysis of time-resolved macromolecular X-ray data. Biophys. J. 84, 2112-2129 (2003).

40. Rajagopal, S., Schmidt, M., Anderson, S., Ihee, H. \& Moffat, K. Analysis of experimental time-resolved crystallographic data by singular value decomposition. Acta Crystallogr. D 60, 860-871 (2004).

41. Kang, Y. et al. Crystal structure of rhodopsin bound to arrestin by femtosecond X-ray laser. Nature 523, 561-567 (2015).

42. Kupitz, C. et al. Structural enzymology using X-ray free electron lasers. Struct. Dyn. 4, 044003 (2017).

43. Olmos, J. L. Jr. et al. Enzyme intermediates captured "on the fly" by mixand-inject serial crystallography. BMC Biol. 16, 59 (2018).

44. Paul, K. et al. Coherent control of an opsin in living brain tissue. Nat. Phys. 13, 1111-1116 (2017).

45. Wang, J. et al. Time-resolved protein activation by proximal decaging in 
living systems. Nature 569, 509-513 (2019).

46. Pandey, S., Bean, R., Sato, T., Mancuso, A. P. \& Schmidt, M. Timeresolved serial femtosecond crystallography at the European X-ray free electron laser. Protocol Exchange, https://doi.org/10.21203/rs.2.14634/v1 (2019).

AQ23

47. Mancuso, A. P., Aquila, A., Borchers, G., Giewekemeyer, K. \& Reimers, N. Technical Design Report: Scientific Instrument Single Particles, Clusters, and Biomolecules (SPB) https://doi.org/10.3204/XFEL.EU/TR-2013-004 (XFEL.EU, 2013).

48. Echelmeier, A. et al. Hydrophobic surface coatings for stable aqueous in oil droplet generation in 3D printed devices. J. Appl. Crystallogr. (in the press, 2019).

AQ24

49. Hutchison, C. D. M. et al. Photocycle populations with femtosecond excitation of crystalline photoactive yellow protein. Chem. Phys. Lett. 654, 63-71 (2016).

50. Nass Kovacs, G. et al. Three-dimensional view of ultrafast dynamics in photoexcited bacteriorhodopsin. Preprint at https://arxiv.org/abs/1905.09002 (2019).

51. Bean, R. J., Aquila, A., Samoylova, L. \& Mancuso, A. P. Design of the mirror optical systems for coherent diffractive imaging at the SPB/SFX instrument of the European XFEL. J. Opt. 18, 074011 (2016).

52. Greiffenberg, D. The AGIPD detector for the European XFEL. J. Instrum. 7, CO1103 (2012).

53. Heisen, B. C. et al. Data Analysis Support in Karabo at European XFEL (JACoW, 2014).

AQ25 
54. Boukhelef, D., Szuba, J., Wrona, K. \& Youngman, C. Software Development for High Speed Data Recording and Processing (JACoW2014).

55. Kirkwood, H. J. et al. Initial observations of the femtosecond timing jitter at the European XFEL. Opt. Lett. 44, 1650-1653 (2019).

56. Mariani, V. et al. OnDA: online data analysis and feedback for serial X-ray imaging. J. Appl. Crystallogr. 49, (1073-1080 (2016).

57. Barty, A. et al. Cheetah: software for high-throughput reduction and analysis of serial femtosecond X-ray diffraction data. J. Appl. Crystallogr. 47, 1118-1131 (2014).

58. Yefanov, O. et al. Accurate determination of segmented X-ray detector geometry. Opt. Express 23, 28459-28470 (2015).

59. Brehm, W. \& Diederichs, K. Breaking the indexing ambiguity in serial crystallography. Acta Crystallogr. D 70, 101-109 (2014).

60. Glownia, J. M. et al. Time-resolved pump-probe experiments at the LCLS. Opt. Express 18, 17620-17630 (2010).

61. Harmand, M. et al. Achieving few-femtosecond time-sorting at hard X-ray free-electron lasers. Nat. Photonics 7, 215-218 (2013).

62. Bionta, M. R. et al. Spectral encoding of X-ray/optical relative delay. Opt. Express 19, 21855-21865 (2011).

63. Murshudov, G. N. et al. REFMAC5 for the refinement of macromolecular crystal structures. Acta Crystallogr. D 67, 355-367 (2011).

64. Winn, M. D. et al. Overview of the CCP4 suite and current developments. Acta Crystallogr. D 67, 235-242 (2011).

65. Ren, Z. et al. A molecular movie at $1.8 \AA$ resolution displays the photocycle of photoactive yellow protein, a eubacterial blue-light receptor, from nanoseconds to seconds. Biochemistry 40, 13788-13801 (2001). 
66. Drenth, J. Principles of Protein X-Ray Crystallography (Springer, 1999)..

67. Terwilliger, T. C. \& Berendzen, J. Bayesian difference refinement. Acta Crystallogr. D 52, 1004-1011 (1996).

68. Emsley, P., Lohkamp, B., Scott, W. G. \& Cowtan, K. Features and development of Coot. Acta Crystallogr. D 66, 486-501 (2010).

69. Nogly, P. et al. Retinal isomerization in bacteriorhodopsin captured by a femtosecond X-ray laser. Science 361, eaat0094 (2018).

70. Richards, F. M. \& Kundrot, C. E. Identification of structural motifs from protein coordinate data - secondary structure and 1st-level supersecondary structure. Proteins 3, 71-84 (1988).

71. Maia, F. R. The coherent X-ray imaging data bank. Nat. Methods 9 , 854-855 (2012).

72. Liang, M. et al. The coherent X-ray imaging instrument at the linac coherent light source. J. Synchrotron Radiat. 22, 514-519 (2015).

73. Graber, T. et al. BioCARS: a synchrotron resource for time-resolved X-ray science. J. Synchrotron Radiat. 18, 658-670 (2011). 